\title{
Activity Recognition for Incomplete Spinal Cord Injury Subjects Using Hidden Markov Models
}

Pichleap Sok

Loyola University Chicago

Follow this and additional works at: https://ecommons.luc.edu/luc_theses

Part of the Computer Sciences Commons

\section{Recommended Citation}

Sok, Pichleap, "Activity Recognition for Incomplete Spinal Cord Injury Subjects Using Hidden Markov Models" (2016). Master's Theses. 3272.

https://ecommons.luc.edu/luc_theses/3272

This Thesis is brought to you for free and open access by the Theses and Dissertations at Loyola eCommons. It has been accepted for inclusion in Master's Theses by an authorized administrator of Loyola eCommons. For more information, please contact ecommons@luc.edu. (c) (i) $\Theta \Theta$

This work is licensed under a Creative Commons Attribution-Noncommercial-No Derivative Works 3.0 License. Copyright @ 2016 Pichleap Sok 
LOYOLA UNIVERSITY CHICAGO

ACTIVITY RECOGNITION FOR

INCOMPLETE SPINAL CORD INJURY SUBJECTS USING

HIDDEN MARKOV MODELS

A THESIS SUBMITTED TO

THE FACULTY OF THE GRADUATE SCHOOL

IN CANDIDACY FOR THE DEGREE OF

MASTER OF SCIENCE

PROGRAM IN COMPUTER SCIENCE

BY

PICHLEAP SOK

CHICAGO, IL

AUGUST 2016 
Copyright by Pichleap Sok, 2016

All rights reserved. 


\section{ACKNOWLEDGEMENTS}

I would like to thank all of the people who made this thesis possible, starting with my wonderful professors in the Department of Computer Science at Loyola University Chicago. Dr. Mark V. Albert gave me the opportunity to work on this fascinating thesis. I still remember the day when we were first discussing the project and potential machine learning techniques to be used in the project. Although machine learning was relatively new to me at that time, I felt so excited. I have enjoyed and cherished every moment working with him: from discussion to code, from proposal to thesis, and from academic advising to career advising. In spite of how busy he was, he put me on his priority list. He has always been a supportive, encouraging, and caring advisor to me. I am extremely grateful to have him as my advisor. This thesis and his Machine Learning course have opened my eyes to the world of machine learning. Dr. Konstantin Laufer has given me invaluable advice and inspiration along the way of my education at Loyola University Chicago. We would spend hours catching up or discussing theories behind programming in his office. I always learned something every time we met. Finally, I would like to thank Dr. Chandra Sekharan for agreeing to be my committee chair and for his invaluable advice. I am very grateful for the help from Dr. Andrew Harrington throughout my years at Loyola University Chicago. He has been the humblest Graduate Program Director I have ever known. I would also like to thank Loyola University Chicago for providing the funds with which to complete my research and writing. 
Many thanks to Asma Mehjabeen for starting off this project as her one-semester Independent Study Project, to Lucas Borges Fernandes and Gabriela Furlan for fixing some bugs in the previous codes, to Mark Rogers and Cam Hudgins for assisting me in finding some relevant technical papers. Last but not least, I would like to thank Arun Jayaraman and his lab ("Rehabilitation Technologies and Outcomes lab") at the Rehabilitation Institute of Chicago for providing data.

My friends in the Department of Computer Science and outside of it have provided me with a much needed cheering section and with necessary periodic distractions during which to regroup and come back to the thesis, refreshed and ready to confront it all over again.

Finally, I would like to extend my gratitude to my dearest family. Without their support, motivation and unconditional love, I would never have made it to where I am today. 


\section{TABLE OF CONTENTS}

ACKNOWLEDGEMENTS

LIST OF TABLES Vi vi

LIST OF FIGURES vii

ABSTRACT viii

CHAPTER I: INTRODUCTION 1

CHAPTER II: BACKGROUND 6

Activity Recognition using Static Machine Learning Classifiers 6

Patient-centered Activity Recognition 9

Markov Models $\quad 12$

Hidden Markov Models $\quad 14$

Hidden Markov Models for Activity Recognition 16

Previous Work $\quad 19$

CHAPTER III: METHODOLOGY 21

Data Collection $\quad 21$

Feature Extraction $\quad 23$

$\begin{array}{ll}\text { Static Classification } & 24\end{array}$

HMM Classification $\quad 27$

Within-Subject Cross Validation 31

Subject-Wise Cross Validation $\quad 32$

Hyperparameter Optimization 33

CHAPTER IV: RESULTS AND DISCUSSION 34

Static Classification Results $\quad 34$

HMM Classification Results $\quad 35$

$\begin{array}{ll}\text { Discussion } & 40\end{array}$

CHAPTER V: CONCLUSION 42

REFERENCES $\quad 43$

$\begin{array}{ll}\text { VITA } & 46\end{array}$ 


\section{LIST OF TABLES}

Table 1. Transition probabilities of the next activity based on the current activity

Table 2. Transition probabilities between activities

Table 3. Features used for activity recognition

Table 4. HMM transition probability matrices

Table 5. Overall accuracy for multiple generative and discriminative classifiers

Table 6. Precision and recall numbers for random forest classifier using within-subject cross validation. Overall precision is $83.3 \%$ and recall is $84.1 \%$

Table 7. Precision and recall numbers for HMM classifier using within-subject cross validation. Overall precision is $87.2 \%$ and recall is $86.9 \%$

Table 8. Classification matrix of random forest and HMM classifier using within-subject 20 -fold cross validation

Table 9. Precision and recall numbers for SVM classifier using subject-wise cross validation. Overall precision is $57.81 \%$ and recall is $56.78 \%$

Table 10. Precision and recall numbers for HMM classifier using subject-wise cross validation. Overall precision is $61.06 \%$ and recall is $62.75 \%$

Table 11. Classification matrix of SVM and HMM classifier using subject-wise cross validation 


\section{LIST OF FIGURES}

Figure 1. The axes of the accelerometer relative to the orientation of the phone

Figure 2. Examples of accelerometer readings of three activities: standing, sitting and walking for a typical user.

Figure 3. Example of accelerometer readings of healthy and Parkinson's patients for three activities: standing, sitting and walking

Figure 4. A graphical representation of a Markov model

Figure 5. Probabilistic parameters of a hidden Markov model

Figure 6. A graphical representation of a HMM for activity recognition

Figure 7. Subjects performed the set of physical activities at home in the order shown 22

Figure 8. Subjects performed the physical activities in the lab in a displayed order that allows every combinations of transitions between activities

Figure 9. The accelerometer readings for different activities

Figure 10. Supervised machine learning classification 26

Figure 11. HMM emission probability

Figure 12. Classification process using hidden Markov models 30

Figure 13. Within-subject 20 -fold cross validation technique

Figure 14. Subject-wise cross validation technique 


\begin{abstract}
Successful activity recognition in patients with motor disabilities can improve patient care by providing researchers and clinicians with valuable information on patient movements and quality of life in real-world settings. Understanding the everyday activities of patients is important for rehabilitation. For researchers, having convenient, objective, and continuous data can drastically improve outcome measures to better compare therapies, and ultimately make recommendations. For clinicians, individual assessment of compliance and outcomes outside the clinic can be more objective, permitting much more tailored recommendations to patients. Most importantly, for individual patients, activity recognition can make this improved health care possible by simply having patients wearing a small sensor, minimizing the need for clinical visits but reaping all the benefits of tailored healthcare.

There are many activity trackers available in the market. But most of them have been designed for healthy subjects. Studies have shown that activity tracking systems designed for healthy subjects can perform poorly on mobility-impaired populations, like those with incomplete spinal cord injury (iSCI) due to their unique patterns of movement. Because iSCI patient populations move in distinct ways, algorithms can and should be specifically tailored for them. By applying machine learning to collect movement data from this specific patient population, we demonstrate how an iSCI-specific system can improve activity recognition with this population.
\end{abstract}


Traditional activity recognition approaches analyze individual clips of accelerometer data to perform activity recognition. These static classifiers are easier to construct, as each clip of data is treated independently, but the structure of events over time is lost. This thesis attempts to improve upon the standard static classification method by augmenting these static classifiers with a dynamic state estimation model —a hidden Markov model (HMM). An HMM takes into account not only the information present in a clip of sensor data, but also the context of that clip over time, which leads to a higher classification accuracy. By using an HMM to go over the predictions made by the static classifier, unlikely sequences of events can be removed and corrected.

Data were collected from thirteen ambulatory incomplete spinal cord injury subjects who were instructed to perform a standardized set of activities while wearing a waist-worn accelerometer in the clinic. Activities included lying, sitting, standing, walking, wheeling, and stair climbing. The accelerometer data was parsed into twosecond clips and a standard set of time-series features were extracted from those clips. Those features were then analyzed by a static classifier to produce probabilistic estimates of the likely activity the subject was performing. Those estimates were then input as observations into the HMM to reclassify ambiguous or improbable sequences of activities made by the static classifier. Multiple classifiers and validation methods were used to assess the ability of the machine learning techniques.

Using within-subject cross validation, static classifiers provided a classification accuracy of $86.3 \%$. By adding another layer of a hidden Markov model, the accuracy improved an additional $2.6 \%$ to $88.9 \%$. In subject-wise cross validation, a hybrid static classifier and HMM model gave the highest classification accuracy of $64.3 \%$, a $1.2 \%$ 
improvement over the model using only static classifiers. Our prediction accuracy was subtle because we dealt with activities that are almost undistinguishable: sitting and wheeling, walking and stair climbing.

Individuals with impaired movements can benefit from improved activity recognition to more objectively, conveniently, and continuously measure patient outcomes. Such measures support therapists, clinicians, and clinical researchers to select the right physical or drug therapies, and further refine selected therapies to improve mobility in patients. 


\section{CHAPTER I}

\section{INTRODUCTION}

Activity tracking plays an important role in the field of rehabilitation, including motivating patients to exercise, allowing clinicians to track patient progress objectively, and for researchers to collect more reliable data to determine what are the best rehabilitation strategies (Arif \& Kattan, 2015). While commercial activity trackers on the market, such as smart watches and wristbands, have surged in popularity in recent years, the use of wearable activity trackers in clinical contexts is still very limited (Lester, Choudhury, \& Borriello, 2006). Product designers of those devices rarely consider patients with impaired movement to be a practical user group; thus, those activity monitoring devices do not work effectively for motor-disability populations.

In the United States, there are more than 8,000 people who suffer from a traumatic spinal cord injury each year (Wirz et al., 2005). After the initial injury, 50\% of motor recovery takes place within two months, and full neurologic recovery takes up to two years of the injury (Wirz et al., 2005). During the process of recovery, the patients undergo treatments that lead to partial functional recovery. While a vast majority of patients indicate that exercise is important for functional recovery, more than half of them either did not have access to exercise equipment or did not have access to a trained therapist to monitor their exercise (Anderson, 2004). With activity trackers, the patients can have the ability to exercise anywhere without traveling to a health care provider, and 
not only do their therapists not have to directly observe them while exercising but also therapists are able to obtain accurate feedback on the quality and quantity of the patients' movements.

The traditional means of evaluating patient mobility are very limited. Patients with motor disabilities, such as those with incomplete spinal cord injury, often must travel to their health care provider or a rehabilitation center to be periodically evaluated and perform a set of exercises, which are overseen by a trained therapist. A trained therapist also must supervise other patients; therefore, he/she must split his/her time among several patients at different locations, or the patients must be grouped together (Lester et al., 2006). The cost of such evaluations, for both patients and therapists, is very time and resource intensive, which makes evaluations difficult to perform frequently. Alternatively, a patient can exercise at home where they have to complete an activity report at the end of the day. Self-reporting, however, is often inaccurate and impractical. Patients may intentionally or unintentionally misreport their activities due to their mental state or forgetfulness. Patients may report more or fewer activities than what they actually accomplish throughout the day (Lester et al., 2006). As an alternative, activity tracking through the use of wearable sensors minimizes expenses and travel while providing objective and continuous measures of a patient's exercise performance (Albert, Toledo, Shapiro, \& Koerding, 2012).

As they become more commercialized, activity tracking devices have become smaller, more affordable, and more available to consumers; they have made a transition from being personal wellness tools to patient-centered clinical tools. Bringing wearable 
activity tracking to populations with movement impairment, however, is particularly challenging. Many consumer-based activity monitors currently available on the market, e.g., Fitbit and Jawbone UP, typically perform limited analyses to estimate step counts, calories, sleep quality, and general activity levels (Chiauzzi, Rodarte, \& DasMahapatra, 2015; Lee, Kim, \& Welk, 2014). They have been designed to track the movement of healthy populations, and therefore not considering the impact of motor impairments that may include muscle weakness, muscle spasms and overactive reflexes. Consumer-based wearable devices for activity tracking are known to not be as accurate when worn by patients with irregular patterns of movement, such as those with incomplete spinal cord injury. In addition to that, the data, generated from the same consumer-based activity tracking devices, is not often integrated into a treatment regimen, thus making it very difficult to be used in a clinical setting (Chiauzzi et al., 2015). Those unresolved obstacles make commercial activity tracking difficult to apply to patients with motor impairments.

Although performing activity recognition in movement-impaired populations is challenging, algorithms can be tailored specifically for populations with unique movement patterns. Activity recognition strategies have been performed for other populations, including individuals with Chronic Obstructive Pulmonary Disease (COPD) (Patel, Mancinelli, Healey, Moy, \& Bonato, 2009), patients with Parkinson’s disease (Albert et al., 2012), and elderly subjects (Najafi, 2003). The studies indicate that their unique movements have a dramatic impact on activity recognition algorithms. These studies also demonstrate that by specifically designing activity recognition strategies for 
those populations, tracking accuracy significantly increased. Consequently, it is indeed possible for activity recognition algorithms to be tailored for unique populations, such as incomplete spinal cord injury patients.

In a recent study on activity recognition in ambulatory subjects with incomplete spinal cord injury, researchers tailored static supervised machine learning techniques to categorize the activities of patients with incomplete spinal cord injury (Albert, Azeze, Courtois \& Jayaraman, 2016). The subjects were instructed to perform a standardized set of movements including lying, sitting, standing, walking, wheeling, and stair climbing. These activities were performed both in the lab and at home while wearing a tri-axial accelerometer around the waist. The data was parsed into ten-second clips. In the same study, they used five different static classifiers: Support Vector Machines, Naïve Bayes, Regularized Logistic Regression, K Nearest Neighbors, and Decision Trees. While this approach yielded promising results, the accuracy is limited as each clip of data is treated independently of the neighboring clips — a significant loss of valuable information for classification.

A hidden Markov model (HMM), on the other hand, has the potential to minimize those misclassifications by combining the dynamic nature of the HMM with the information provided by a static classifier. As the HMM considers activities over a long timeframe, it will be able to leverage the predictions made by the static classifier and make the most logical predictions based on observations. Hidden Markov models are frequently used to classify various activities with regular rhythms, such as speech recognition, handwriting, gesture recognition, and activity recognition (Gaikwad, 2012). 
After taking advantage of the relative strengths of these classification paradigms, improvements in speech and activity recognition were statistically significant (Ganapathiraju, Hamaker, \& Picone, 2000; Lester, Choudhury, Kern, Borriello, \& Hannaford, 2005). Similar improvements in performance were found when using the hybrid model of a static classifier (SVM) with an HMM on activity tracking for Parkinson's populations (Antos, Albert, \& Kording, 2014).

In this research study, we attempt to improve upon the standard static classification method for accelerometer-based activity recognition in the context of activity tracking for people with incomplete spinal cord injury. We will be augmenting standard machine learning classifiers with an HMM to further improve the prediction accuracy as compared to using only static classifiers. This technique will be applied to movement data from subjects with incomplete spinal cord injury, which was acquired through the Rehabilitation Technologies and Outcomes lab directed by Arun Jayaraman, PT/Ph.D. at the Rehabilitation Institute of Chicago (RIC). 


\section{CHAPTER II}

\section{BACKGROUND}

In this chapter the intent is to establish an understanding of activity recognition, hidden Markov models and previous work. The first section presents activity recognition using a standard machine learning approach through static classifiers, followed by activity recognition tailored for specific patient populations. The later sections introduce Markov models, laying the foundations for the concepts in hidden Markov models (HMMs) in the following section. Upon this foundation, insight and previous work in using hidden Markov models for activity recognition will be presented.

\section{Activity Recognition using Static Machine Learning Classifiers}

Activity recognition aims to predict human activities, particularly those that are likely to occur in real-life settings. Successful activity recognition can lead to many potential applications, especially in the health care field. Although accurate activity recognition is particularly challenging because human activities are complex and highly diverse, there has been significant improvement in predicting human activity in recent years.

As accelerometers in consumer products, including smart phones, have become widely available and cheap, the use of accelerometers in activity recognition has gained more attention. The latest generation of smart phones contain tri-axial accelerometers that

measure acceleration along the $\mathrm{x}, \mathrm{y}$ and $\mathrm{z}$ axes (Figure 1). The ability to detect orientation 
of the device provides useful information for activity recognition (Ravi, Dandekar, Mysore, \& Littman, 2005; Kwapisz, Weiss, \& Moore, 2011).

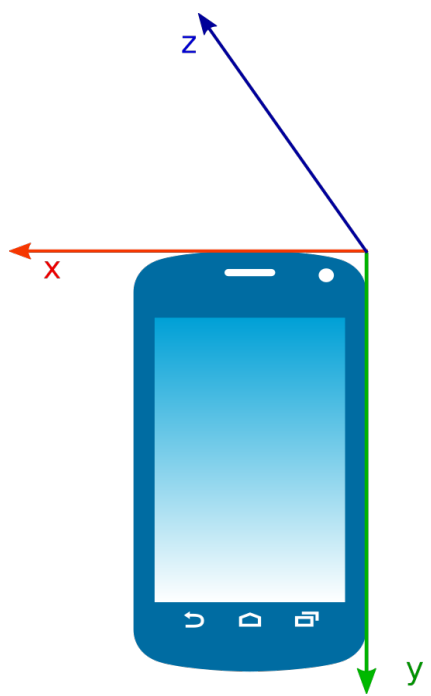

Figure 1. The axes of the accelerometer relative to the orientation of the phone.

Data collected from accelerometer sensors is used to train standard supervised machine learning classifiers in an attempt to recognize/predict human activities. Even though some pairs of activities are particularly difficult to distinguish based solely on accelerometer readings, such as standing and sitting for a waist-worn sensor, as shown in Figure 2, standard machine learning classifiers are generally quite capable of recognizing human activities from accelerometer data with fairly high accuracy.

In previous work on activity recognition, Ravi et al. (2005) attempted to recognize human activities using accelerometer data. Subjects had an accelerometer-enabled device worn on the waist while performing activities, which include standing, walking, running, climbing up stairs, climbing down stairs, doing sit-ups, vacuuming, and brushing teeth. With 10-fold cross-validation for each classifier, and using only static classifiers, such as Naïve Bayes (NB), Support Vector Machines (SVM), K Nearest Neighbors, and 


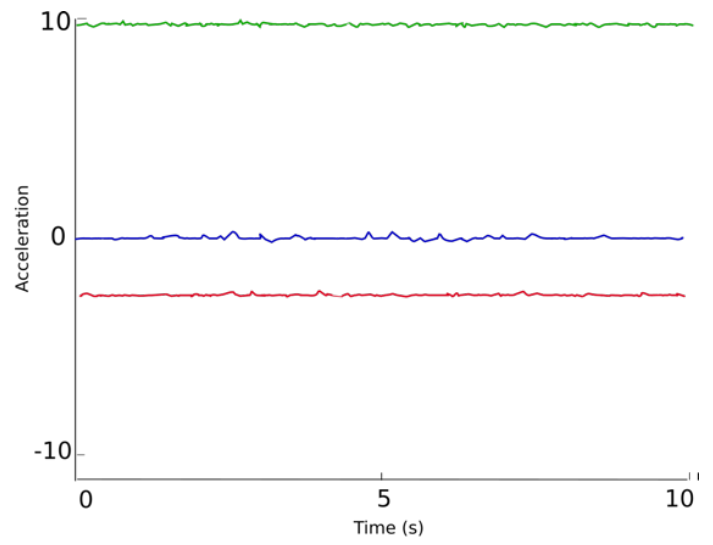

(a) Standing

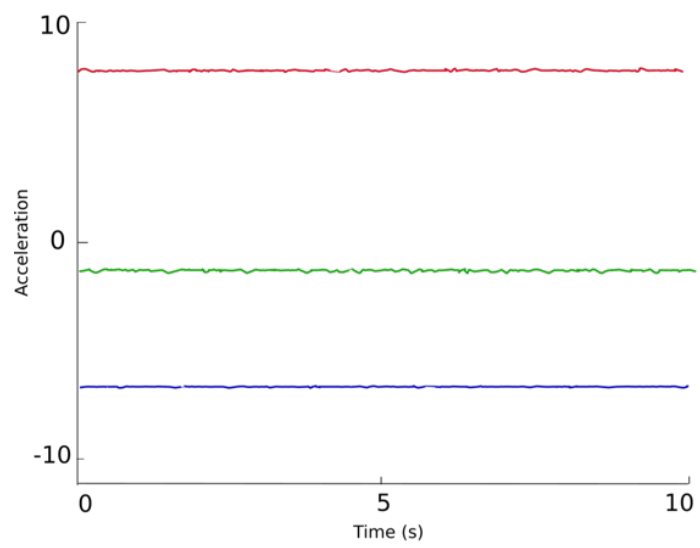

(b) Sitting

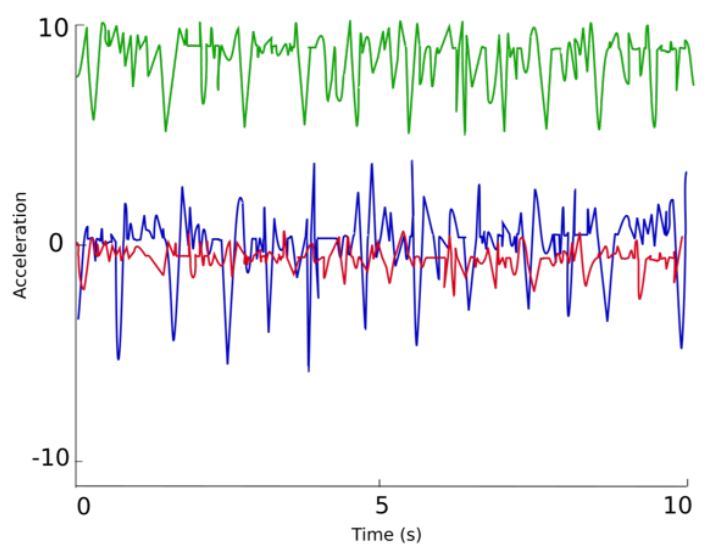

(c) Walking

Figure 2. Examples of accelerometer readings for three activities: standing, sitting and walking for a typical user.Red, green, and blue lines are the $x, y$, and z-axis accelerations. 
Decision Trees, more than $90 \%$ accuracy was achieved (Ravi et al., 2005). Kwapisz et al. (2011) also conducted a similar activity recognition experiment, where twenty-nine volunteer subjects carried an Android phone in their front pants leg pocket while performing certain activities, including walking, jogging, ascending stairs, descending stairs, siting, and standing for specific periods of time. In most cases, they achieved high levels of overall accuracy.

\section{Patient-centered Activity Recognition}

Activity recognition has the potential to better inform patient care by offering patients and their healthcare providers a real-time assessment of their activities. The evaluation is useful, especially for patients with motor disabilities, in quantifying levels of everyday movements and their symptoms both in the clinic and at home (Albert, Toledo, Shapiro, \& Koerding, 2012). The patient-centered activity recognition strategies, however, are much more challenging since subjects with ambulatory impairments move in significantly different ways from healthy subjects.

There have been a large number of activity recognition systems designed for and tested on healthy subjects. However, many approaches that perform well on healthy subjects may perform poorly on populations with impaired mobility that move in unique ways. Albert et al. (2012) applied accelerometer-enabled activity recognition in populations with Parkinson's Disease (PD). Data was collected on both PD subjects and healthy subjects. The study participants, both healthy and Parkinson's subjects, were asked to carry phones with a standard built-in tri-axial accelerometer in their front pockets while performing a series of activities. While they achieved fairly high accuracy 
on healthy subjects, the accuracy level significantly dropped from $92.2 \%$ to $60.3 \%$ when the model trained with healthy subject data was applied to Parkinson's subjects. However, the researchers achieved significantly better accuracy when the classification model was cross validated on Parkinson's participants - though still not as high in comparison to the accuracy achieved with healthy participants. Figure 3, adapted from the same work of Albert et al (2012), demonstrates some differences in accelerometer readings between Parkinson's patients and healthy subjects for three activities: standing, sitting and walking. This indicates the necessity of taking into account the population's symptoms and their movement patterns when designing the activity recognition algorithm.

Previous studies show the possibility of classifying human activities for specific patient populations with reasonably high accuracy using standard machine learning classifiers. Many previous approaches, however, are best at recognizing activities that involve significant changes in acceleration, such as jogging and walking (Kwapisz et al., 2011). But, they work poorly at classifying activities with accelerometer readings that present subtle distinctions, such as standing and sitting. This task is even more challenging for patients with movement impairment, adding additional, uncharacteristic motion to the activities. Combining those classifiers with a hidden Markov model, on the other hand, can significantly increase the accuracy of activity recognition (Lester, Choudhury, Kern, Borriello, \& Hannaford, 2005; Ganapathiraju, Hamaker, \& Picone, 2000; Antos, Albert, \& Kording, 2014). Before presenting the description of a hidden 

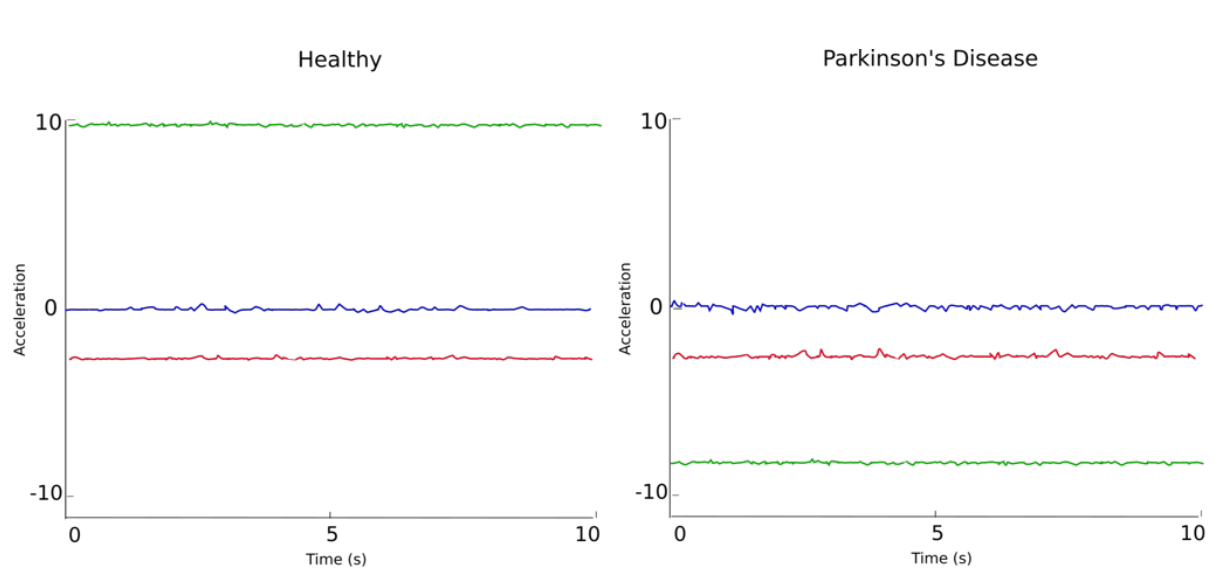

(a) Standing

Healthy
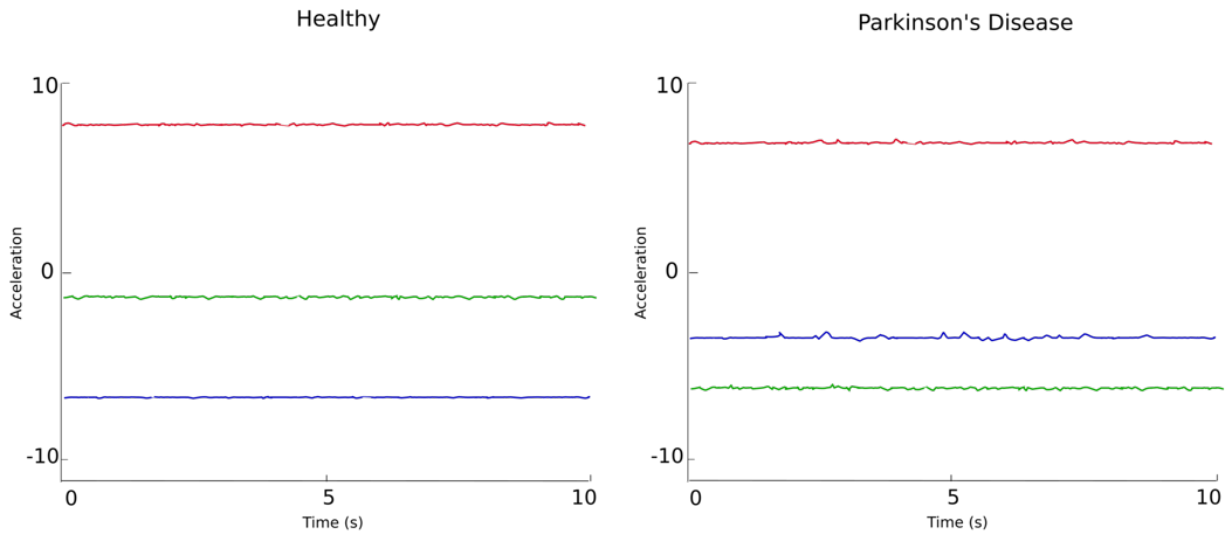

(b) Sitting
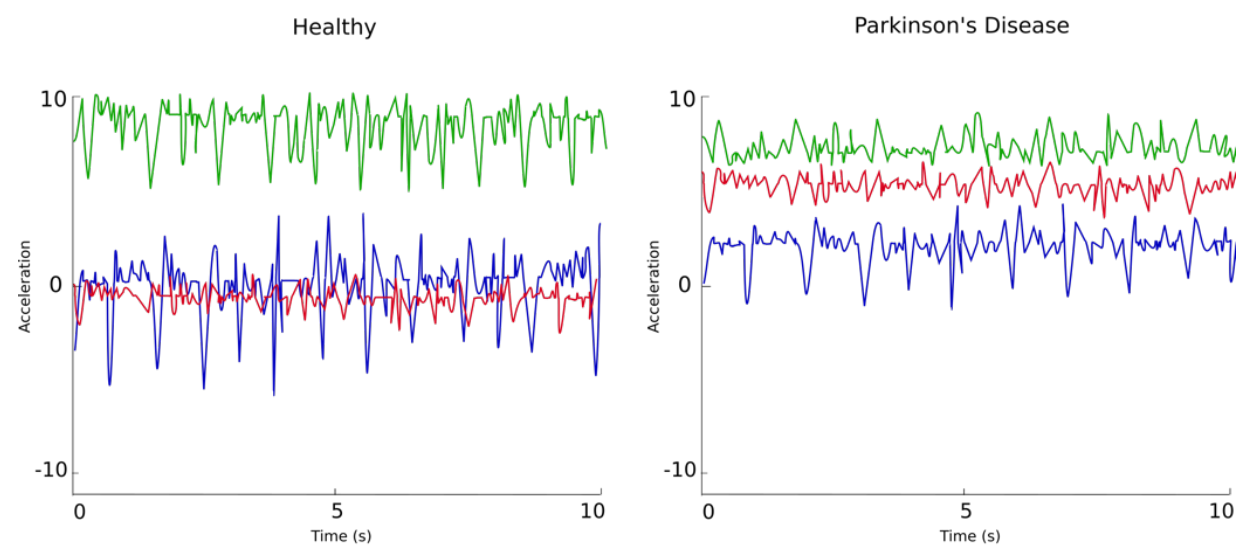

(c) Walking

Figure 3. Example of accelerometer readings of healthy and Parkinson's patients for three activities: standing, sitting and walking. Adapted from "Using mobile phones for activity recognition in Parkinson's patients, ” by Albert, Toledo, Shapiro and Koerding Author, 2012, Frontiers in Neurology, 3, p. 4. Copyright (C) 2012 Albert, Toledo, Shapiro and Koerding. 
Markov model, the observed Markov model, upon which HMMs are based, is presented.

\section{Markov Models}

A Markov model, also known as a Markov process, is based on the concept of "memorylessness." In other words, it is a stochastic model in which the future state is only dependent on the current state, not the past states. A first-order Markov assumption is that the probability of a current event at time $n$, given all past and present events, only depends on the most recent event proximal to time $n-1$ (Fosler-Lussier, 1998). In a sequence $\left\{w_{1}, w_{2}, w_{3}, \ldots, w_{n}\right\}$ :

$$
P\left(w_{n} \mid w_{n-1}, w_{n-2} \ldots, w_{1}\right)=P\left(w_{n} \mid w_{n-1}\right)
$$

In a classic Markov model, states are directly visible to observers. Therefore, the only parameters of the model are the transition probabilities. The transition probability is the chance of moving from one state to another at each point in time. In the context of activity recognition, Figure 4 demonstrates a simple Markov model with three states: standing, sitting and walking. By knowing which state someone is currently at, we can make probabilistic estimates on how long they will remain in the current state, and when they may transition to other states if we know which states they are likely to transition to.

Let's assume we know the probability of someone remaining in the same state and the transition probability that someone is likely to transition from one state to another, as shown in Figure 4. The states are activities in this context. We know the current activity of the person. We are now trying to observe the next activity that he/she will be doing, given the transition probabilities as shown in Table 1. Assuming that the 
current activity is sitting, the probability that the next activity will be sitting and the activity after that one will be standing can be translated into:

$$
\begin{aligned}
P\left(\mathrm{a}_{2}=\text { Sitting, } \mathrm{a}_{3}=\text { Standing } \mid \mathrm{a}_{1}=\text { Sitting }\right)= & P\left(\mathrm{a}_{3}=\text { Standing } \mid \mathrm{a}_{2}=\text { Sitting, } \mathrm{a}_{2}=\text { Sitting }\right) * \\
& P\left(\mathrm{a}_{2}=\text { Sitting } \mid \mathrm{a}_{1}=\text { Sitting }\right) \\
= & P\left(\mathrm{a}_{3}=\text { Standing } \mid \mathrm{a}_{2}=\text { Sitting }\right) * \\
& P\left(\mathrm{a}_{2}=\text { Sitting } \mid \mathrm{a}_{1}=\text { Sitting }\right) \\
= & (0.19) *(0.8) \\
= & 0.152
\end{aligned}
$$

The fundamental limitation of the Markov model is that, in the real world, the states are not always known, so inferences that can be made assuming the state is known are not as helpful. To handle this situation, we use what is referred to as a hidden Markov model.

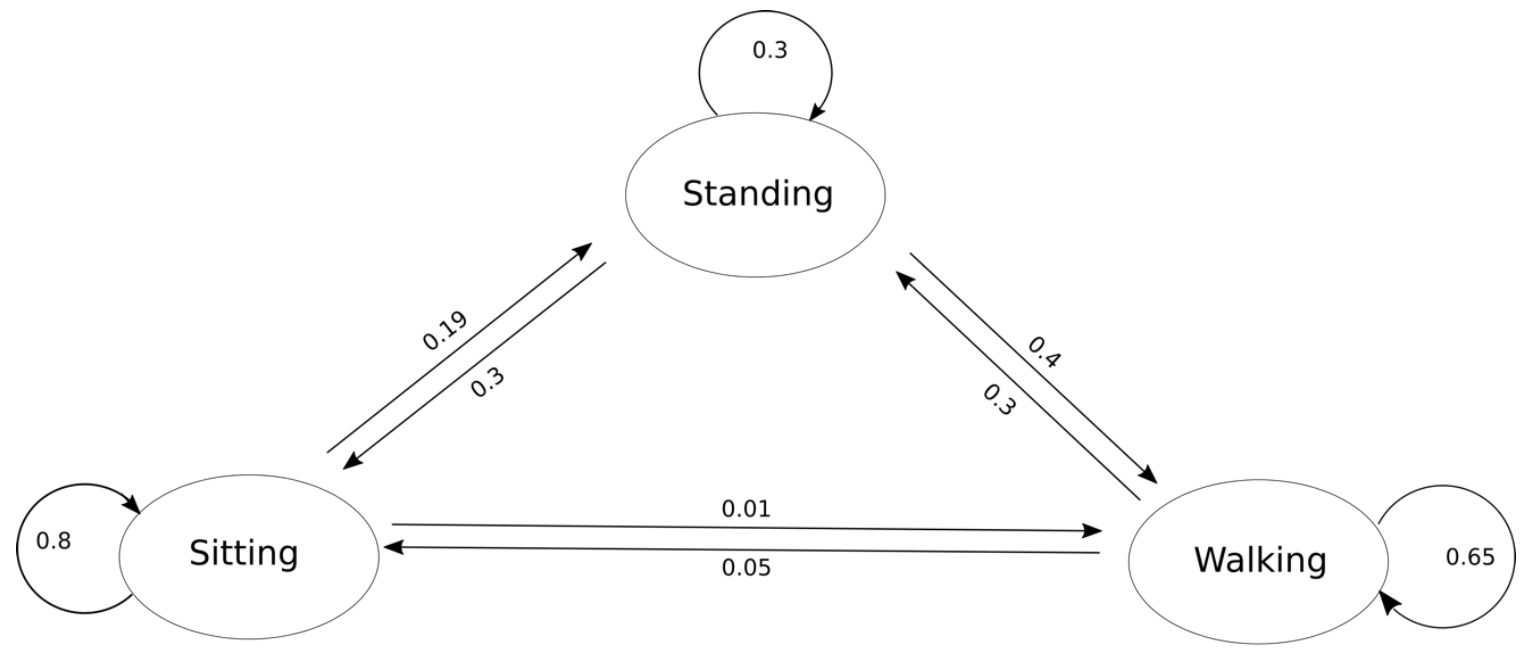

Figure 4. A graphical representation of a Markov model. 


\begin{tabular}{|c|c|c|c|c|}
\cline { 2 - 5 } \multicolumn{2}{c|}{} & \multicolumn{3}{c|}{ Next Activity } \\
\cline { 2 - 5 } & Standing & Sitting & Walking \\
\hline \multirow{2}{*}{} & Standing & 0.3 & 0.3 & 0.4 \\
\cline { 2 - 5 } & Sitting & 0.19 & 0.8 & 0.01 \\
\cline { 2 - 5 } & Walking & 0.3 & 0.05 & 0.65 \\
\hline
\end{tabular}

Table 1. Transition probabilities of the next activity based on the current activity.

Hidden Markov Models

A hidden Markov model (HMM) is a Markov model with unobserved (hidden) states. Hidden Markov models are frequently used to classify various activities with temporal structure such as speech, handwriting, part-of-speech tagging and gesture recognition (Gaikwad, 2012). The main goal of the model is to find the hidden states from observable data. Unlike in a regular Markov model, the state of the data in a hidden Markov model is not directly visible to an observer, but observations related to each state are visible. Observations are probabilistically related to their (hidden) states, and so the states themselves can be inferred from the observations. In other words, the sequence of observations allows us to make probabilistic inferences about the sequence of the hidden states (Gaikwad, 2012; Kim, Helal, \& Cook, 2010).

As illustrated in Figure 5, a hidden Markov model consists of two main parameters: transition probabilities and observation/emission probabilities. Given the observed output sequence $\left(y_{1}, y_{2}, y_{3}\right)$, one use of an HMM is to determine the hidden state sequence $\left(x_{1}, x_{2}, x_{3}\right)$. In order to accomplish that, HMM requires two independent assumptions. 
The first assumption refers to a first-order Markov assumption as described in the Markov Models section. The assumption states that the future state depends only on the current state. That is, at time $t$, the hidden state $x_{t}$, is conditionally independent of the past states, but dependent only on the previous hidden state $x_{t-1}$ (Kim et al., 2010).

$$
P\left(x_{t} \mid x_{2} \ldots, x_{t-1}\right)=P\left(x_{t} \mid x_{t-1}\right)
$$

Another HMM assumption is that observation probabilities are conditionally independent of all other past states and observable variables, given the current hidden state $x$. In other words, the observable variable at time $t, y_{t}$, depends only on the current hidden state $x_{t}$ (Kim et al., 2010).

$$
P\left(y_{t} \mid x_{t}, y_{1}, y_{2} \ldots, y_{t-1}, x_{1}, x_{2} \ldots, x_{t-1}\right)=P\left(y_{t} \mid x_{t}\right)
$$

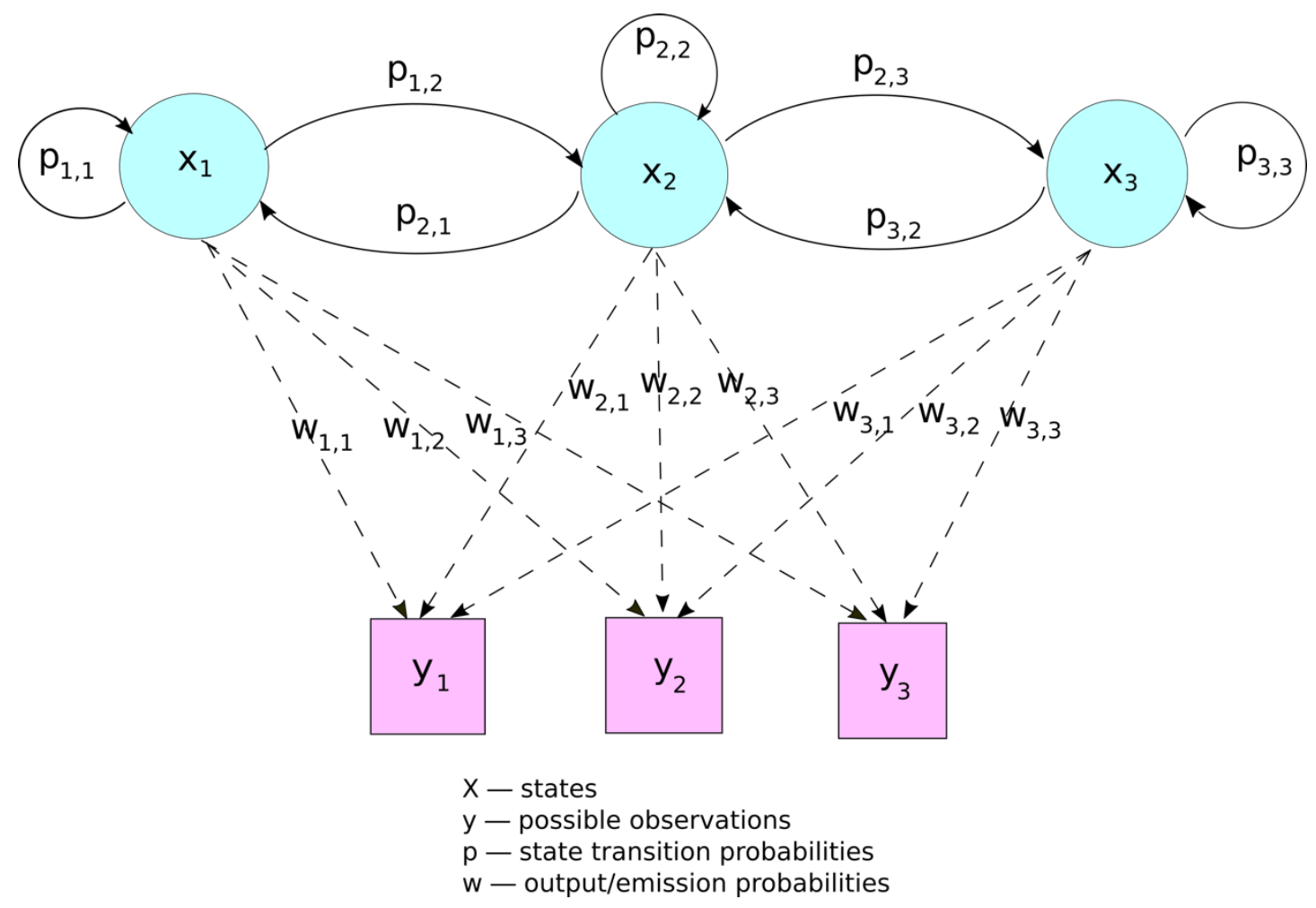

Figure 5. Probabilistic parameters of a hidden Markov model. 
Therefore, to find the probability of a hidden state sequence $\left(x_{1}, x_{2}, \ldots, x_{t}\right)$ from an observed output sequence $\left(y_{1}, y_{2}, \ldots, y_{t}\right)$, an HMM finds the probability that outcome $y_{t}$ is observed in state $x_{t}$, which is a joint probability $P(y, x)$ of the transition probability $P\left(x_{t} \mid x_{t-1}\right)$ and the observation probability $P\left(y_{t} \mid x_{t}\right)$ (Kim et al., 2010).

$$
P(y, x)=\prod_{t-1}^{T} P\left(x_{t} \mid x_{t-1}\right) P\left(y_{t} \mid x_{t}\right)
$$

\section{Hidden Markov Models for Activity Recognition}

Markov models can accurately model simple states and transitions, but when activities are complex or unfamiliar, it is often difficult to fit an appropriate Markov model. Fortunately, a hidden Markov model allows us to indirectly build a model of activities by observing signals from complex or unfamiliar activities (Kim et al., 2010). Through investigating the effect of the activities on our observations, a hidden Markov model is able to model sequential data in activity recognition.

Figure 6 illustrates an HMM which is used in the context of activity recognition. Hidden states are standing, running, and walking. In the hidden Markov model approach, those states are not obvious to an observer who may only have access to sensor readings. Transition probabilities between states are shown in Table 2 . The possibility that a person remains in the same state is generally higher than the possibility that he/she transitions to any other states. Those numbers can be measured or estimated by investigating how often the person changes from one state to another. The states "Low", "Medium" and "High" are activity levels or average acceleration changes in accelerometer data. They are the observable output of the model. Based on the transition probabilities and the output 
sequence of the observations, we can mathematically infer the most logical activity of those hidden states.

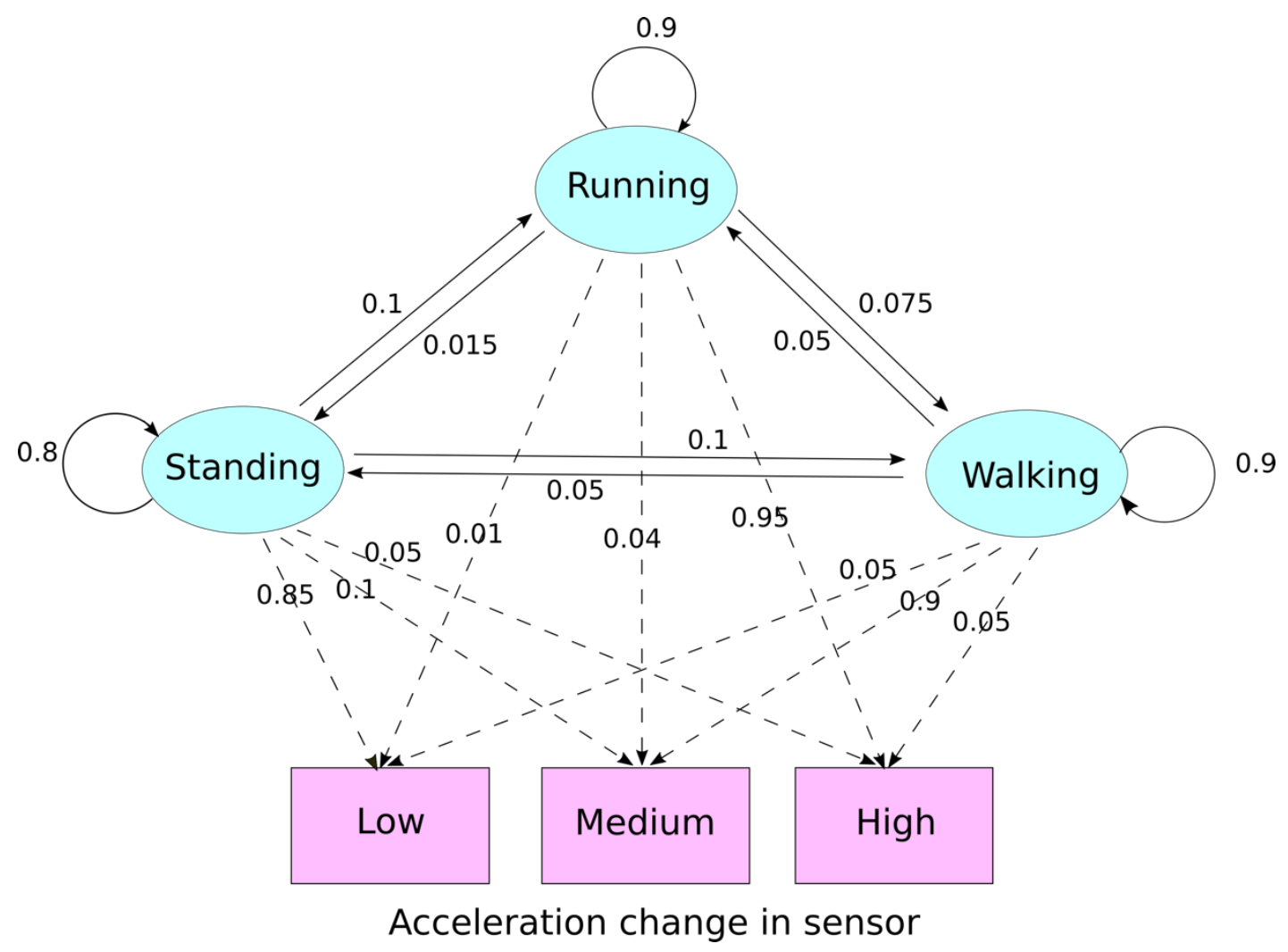

Figure 6. A graphical representation of a HMM for activity recognition.

\begin{tabular}{|c|c|c|c|c|}
\cline { 3 - 5 } \multicolumn{2}{c|}{} & \multicolumn{3}{c|}{ To Activity } \\
\cline { 2 - 5 } \multicolumn{2}{c|}{} & Standing & Running & Walking \\
\hline \multirow{3}{*}{} & Standing & 0.8 & 0.1 & 0.1 \\
\cline { 2 - 5 } & Running & 0.015 & 0.9 & 0.075 \\
\cline { 2 - 5 } & Walking & 0.05 & 0.05 & 0.9 \\
\hline
\end{tabular}

Table 2. Transition probabilities between activities.

HMMs have been successfully used in modeling different types of time-series data, e.g. speech recognition, gesture recognition, and activity recognition. Instead of 
using only standard static machine learning classifiers, Lester et al. (2005) used a hybrid approach, combining static supervised machine learning classifiers with HMMs for modeling human activities. He used the results of static classifiers as input for HMMs. That is, the posterior probabilities of static classifiers were used as observations to train HMMs. By using the posterior probabilities, we can take advantage of the results from the discriminatively trained classifier, as well as reduce the complexity of the HMMs (Lester et al., 2005). Naïve Bayes and Decision Stumps were used as static classifiers in their experiment. Although results from both static classifiers were accurate on their own, there are some scattered misclassifications. With an additional HMM layer on top of static classifiers, the accuracy was slightly improved.

In another experiment on activity recognition, twelve subjects were prompted to perform a sequence of activities: walking, sitting, standing and periodically changing their phones' location (Antos et al., 2013). The researchers examined the accuracy of activity tracking when the phone was placed in different locations and orientations. Using SVMs + HMMs to track activities and phone locations, accuracy improved as compared to using only SVMs. Due to temporal irregularities from spastic movements or occasional bouts of tremor, the use of SVM static classifiers alone can often lead to misclassifications. HMMs, on the other hand, help smooth out those misclassifications by inferring the most logical activities based on neighboring activities when ambiguity arises. The study shows the combination of SVMs with HMMs provides a more robust approach to activity recognition. 


\section{Previous Work}

Taking advantage of the temporal integration of HMMs and the high immediate discriminative power of static machine learning classifiers, we will extend the previous work that used solely static classifiers to perform activity recognition on incomplete spinal cord injury patients. In that study, data was collected both in-lab and at-home with subjects performing a standardized set of activities: lying, sitting, standing, walking, wheeling and stair climbing. Activity-labeled ten-second clips were extracted, and a standard set of time-series features were automatically selected, weighed, and combined to maximize classification accuracy. They used five different static classifiers: Support Vector Machine (SVM), Naïve Bayes, Regularized Logistic Regression, K Nearest Neighbors, and Decision Trees (Albert, Azeze, Courtois \& Jayaraman, 2016).

Consequently, SVMs yielded the most promising result with $91.2 \%$ accuracy for in-lab activities and $85.6 \%$ accuracy for at-home activities using within-subject 10 -fold cross-validation. Note, however, that these accuracy measures are averages with some pairs of activities, such as standing vs sitting, and walking vs stair climbing, are more likely to be misclassified due to their similarities on accelerometer readings.

One significant drawback in using static classifiers, such as SVMs, is that they are not sufficient to classify activities that could be easily misread on accelerometer readings, such as sitting vs standing. A static classifier analyzes a clip of data independently, without taking into consideration either pre- or post-activities. This approach is problematic when some pairs of activities are similar on accelerometer readings but easily to interpret by context. By classifying those clips independently and ignoring the 
fact that people's actions are extended over time, this static classification method is prone to errors. An example of this would be an individual lying down for a long period of time then rolling over while continuing to lie down. An SVM without a "rolling over" class would likely classify the data during rolling over as an action, such as standing up or walking, then would continue to predict that the individual is laying down. To eliminate such problems, Lester et al. (2005) have shown that adding an HMM layer on top of the static classification can lead to more accurate predictions.

In this research study, we take advantage of both static and dynamic classifiers by combining static classifiers with HMMs, and applying them to the same dataset collected from incomplete spinal cord injury patients to further improve prediction accuracy. Improved activity recognition can improve clinical assessments in individuals, and provide valuable data to validate and refine therapies to improve mobility in patients. 


\section{CHAPTER III \\ METHODOLOGY}

This chapter chronologically follows the sequence of events necessary to complete the study. First, the data is collected, features are extracted from the data, and the static classification stage is described. The way the HMM approach is specifically used is presented, and the various validation methods are presented to assess its accuracy in different contexts.

\section{Data Collection}

In previous studies, researchers use supervised static machine learning classifiers, including Support Vector Machines (SVMs), Naïve Bayes, Regularized Logistic Regression, K Nearest Neighbors, and Decision Trees, to perform in-lab and at-home activity recognition for incomplete spinal cord injury patients (Albert, Azeze, Courtois \& Jayaraman, 2016). To further improve the accuracy, as well as to show how good hidden Markov models are in smoothing out the misclassifications produced by the static machine learning classifiers, we apply the static classifier-HMM hybrid approach to the same dataset that was used in their experiment.

The data set was collected from thirteen ambulatory incomplete spinal cord injury subjects - nine males and four females with an age range from 22 to 50 . They performed a series of activities with an accelerometer worn on their waists. Subjects were instructed 
to carry out the same activity set twice: in the lab and then at home. At home, subjects had to perform the following activities: lying, sitting, walking, standing, wheeling, and

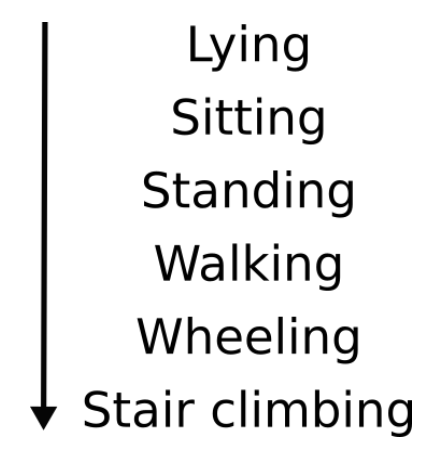

Figure 7. Subjects performed the set of physical activities at home in the order shown.

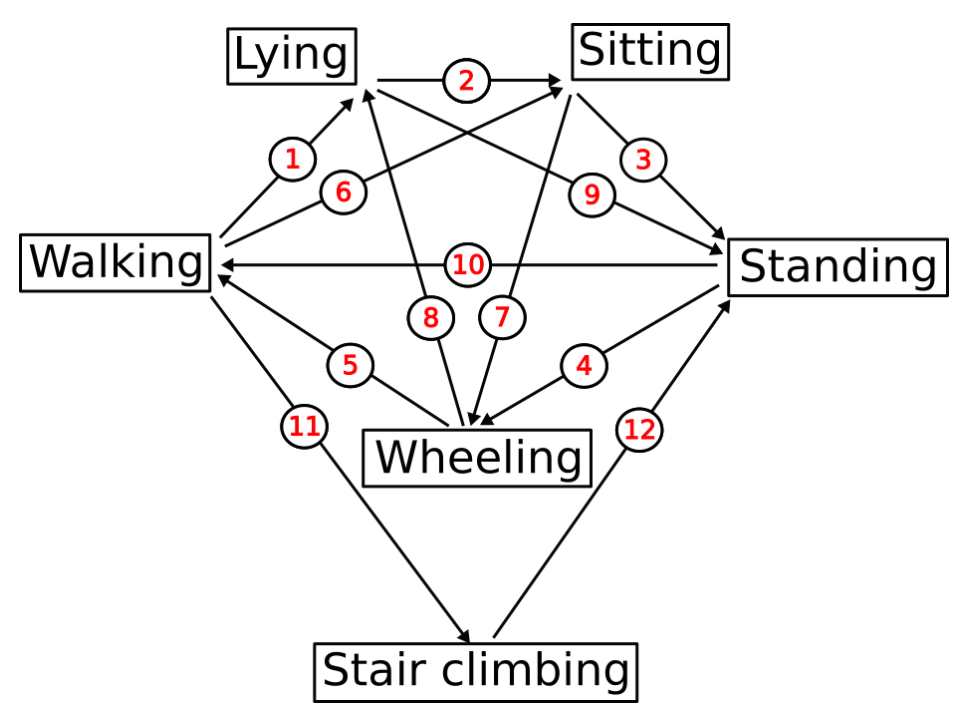

Figure 8. Subjects performed the physical activities in the lab in a displayed order that allows every combination of transitions between activities. Adapted from "In-lab versus at-home activity recognition in ambulatory subjects with incomplete spinal cord injury" by Albert, Azeze, Courtois and Jayaraman Author, 2016, submitted. 
stair climbing in a sequence shown in Figure 7. In the lab, as illustrated in Figure 8, subjects were asked to do the same activity set in an order that allows every combination of transitions between activities, except stair climbing due to accessibility of the stairs.

The accelerometer data has the following attributes: time and acceleration on each axis. Movement data was recorded using an Actigraph wGT3X tri-axial accelerometer worn on subjects' waists using a provided waist strap. An in-house developed MATLAB GUI was used to label the recorded accelerometer data (Figure 9). The accelerometer sampled at a rate of 100 Hertz with a dynamic range of $+/-8$ g's.

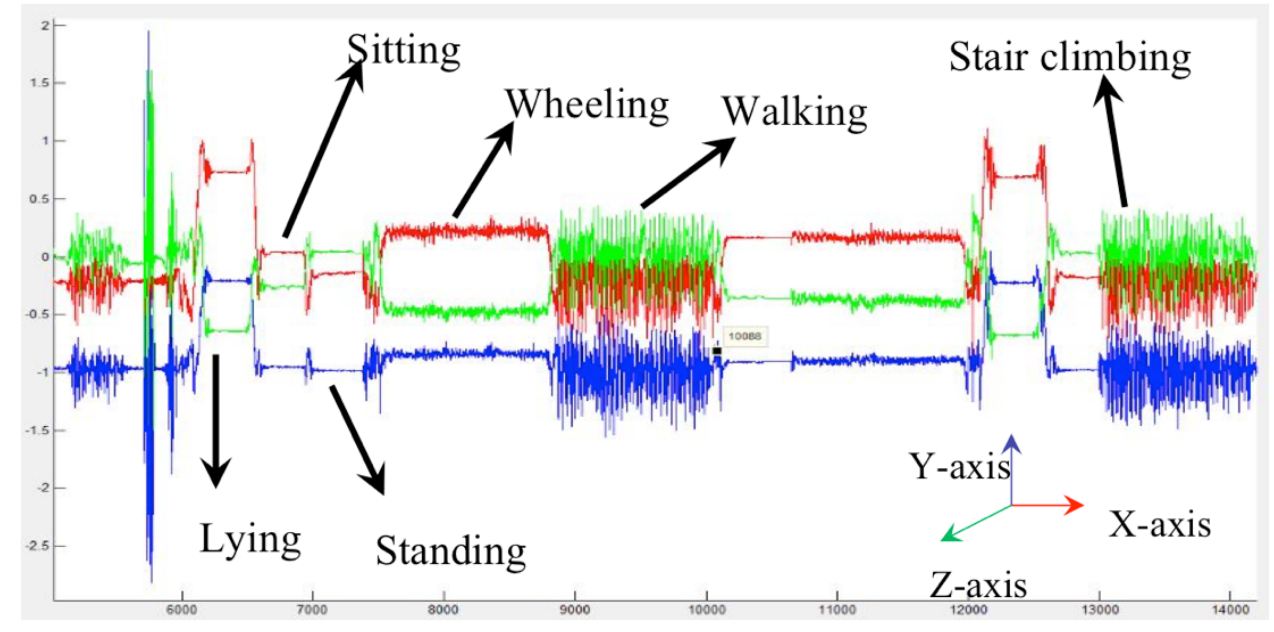

Figure 9. The accelerometer readings for different activities.

\section{Feature Extraction}

Activities were visually identified based on the acceleration values on a specific axis and the expected temporal order of the instructed activities. The accelerometer signals were then segmented into two-second clips, and a series of features were extracted from each of those data clips. 
Thirty seven features were extracted from each clip of the tri-axial accelerometer.

The extracted features were shown in Table 3. Those features have been proven effective in prior work (Albert, Toledo, Shapiro, \& Koerding, 2012; Albert, Azeze, Courtois, \& Jayaraman, 2016). Among those features, standard machine learning classifiers automatically select, weigh, and combine a standard set of time-series features on these clips.

\begin{tabular}{|l|c|}
\hline \multicolumn{1}{|c|}{ Description } & $\begin{array}{c}\text { Total number } \\
\text { of values }\end{array}$ \\
\hline Mean: meanX, meanY, meanZ & 3 \\
Absolute value of the mean & 3 \\
Moments: standard deviation, skew, kurtosis & 9 \\
Root mean square & 3 \\
Extremes: min, max, abs min, abs max & 12 \\
Cross product means: xy, xz, yz & 3 \\
Absolute mean of the cross products & 3 \\
Overall mean acceleration & 1 \\
\hline Total & $\mathbf{3 7}$ \\
\hline
\end{tabular}

Table 3. Features used for activity recognition.

\section{Static Classification}

Although there are many supervised machine learning algorithms, Figure 10 shows the basic steps to obtain a prediction model, from data preprocessing to fitting and testing a model for predictions. In a supervised machine learning algorithm, there are two major parts: training and testing. Training data includes both the input and the desired results, while test data is used for prediction. When the true class of the test data is known, it can be used to evaluate the effectiveness of the model. Sets of time-series 
features were then extracted from each data set. In this research study, we consider the following static classifiers for the static classification algorithm.

\section{Support Vector Machines (SVM)}

2. Naïve Bayes

3. Regularized Logistic Regression

4. K Nearest Neighbors

5. Decision Trees

6. Random Forest

The output of the static classifiers are posterior probabilities which we use as input for the HMM classifier later on. A static classifier predicts the label for each data clip independently. This independent assumption may be invalid, but the outcome of this assumption can help with the HMM classification. "A temporal model that uses the confidence of predictions from previous classifiers instead of using raw features is likely to have greater impact on the performance" (Lester, Choudhury, Kern, Borriello, \& Hannaford, 2005). The HMM model classifies sequences of activities with consideration to how people transition between activities. In the next section, we describe how we combine the results of static supervised machine learning classifiers to build time-series activity recognition models 


\section{(a) Training}

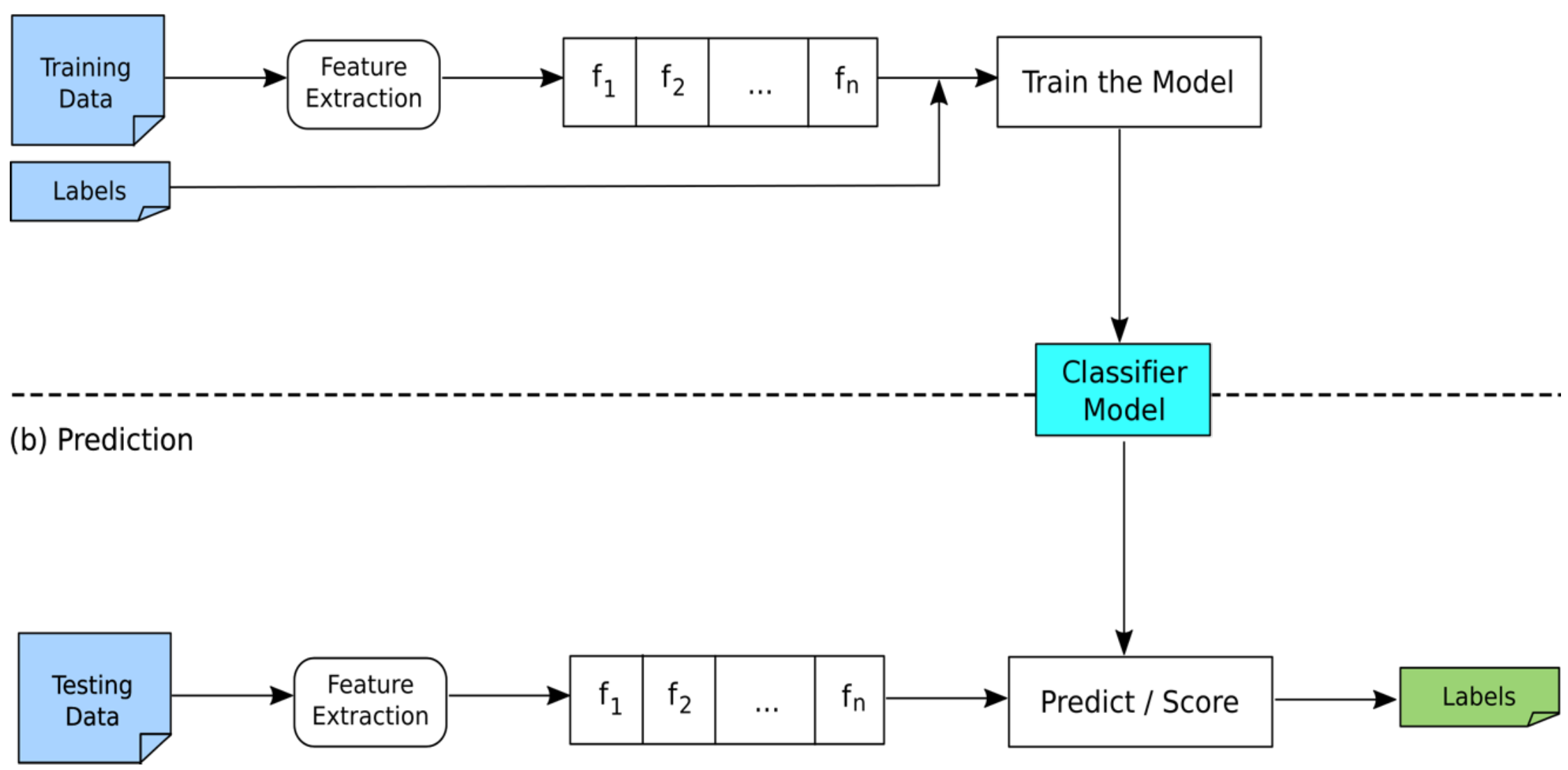

Figure 10. Supervised machine learning classification. 


\section{HMM Classification}

Hidden Markov models have been successfully used in modeling different types of sequential pattern recognition tasks, such as speech recognition, gene prediction, gesture recognition, and activity recognition. Although HMMs can work with raw signals

directly, they are more effective when applied to features that are expected to improve recognition accuracy. In our case, our HMMs use posterior probabilities of static classifiers as shown in Figure 11. In other words, HMMs use output from the static classifiers as input to reclassify the probabilistic inferences made by the static classifiers. The entire classification process using hidden Markov models on top of static classification is illustrated in Figure 12.

To create an HMM model, there are two important parameters: the state transition matrix and the emission probability matrix. All the parameters were set to be uniform across all analyses. A transition matrix, as shown in Table 4, was calculated by determining the probability from hidden state $R_{i}$ to state $R_{j}$, which results in the following transition matrix $P$ wherein indexes one to six indicate the following states, respectively: lying, sitting, stair climbing, standing, walking, or wheeling.

$$
P=\left[\begin{array}{cccc}
P_{1,1} & P_{1,2} & & P_{1,6} \\
P_{2,1} & P_{2,2} & \cdots & P_{2,6} \\
& \vdots & \ddots & \vdots \\
P_{6,1} & P_{6,2} & \cdots & P_{6,6}
\end{array}\right]
$$




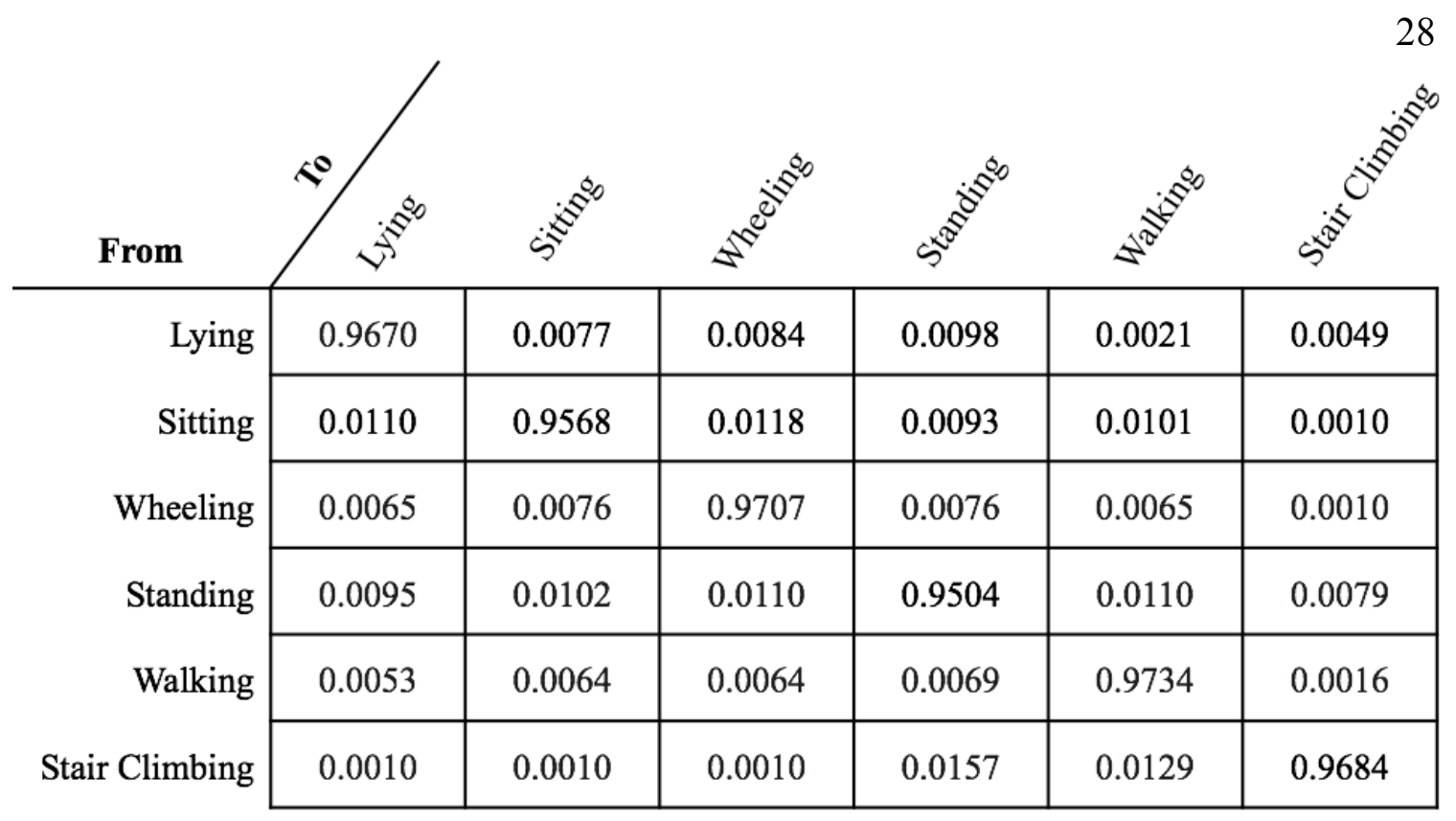

Table 4. HMM transition probability matrices.

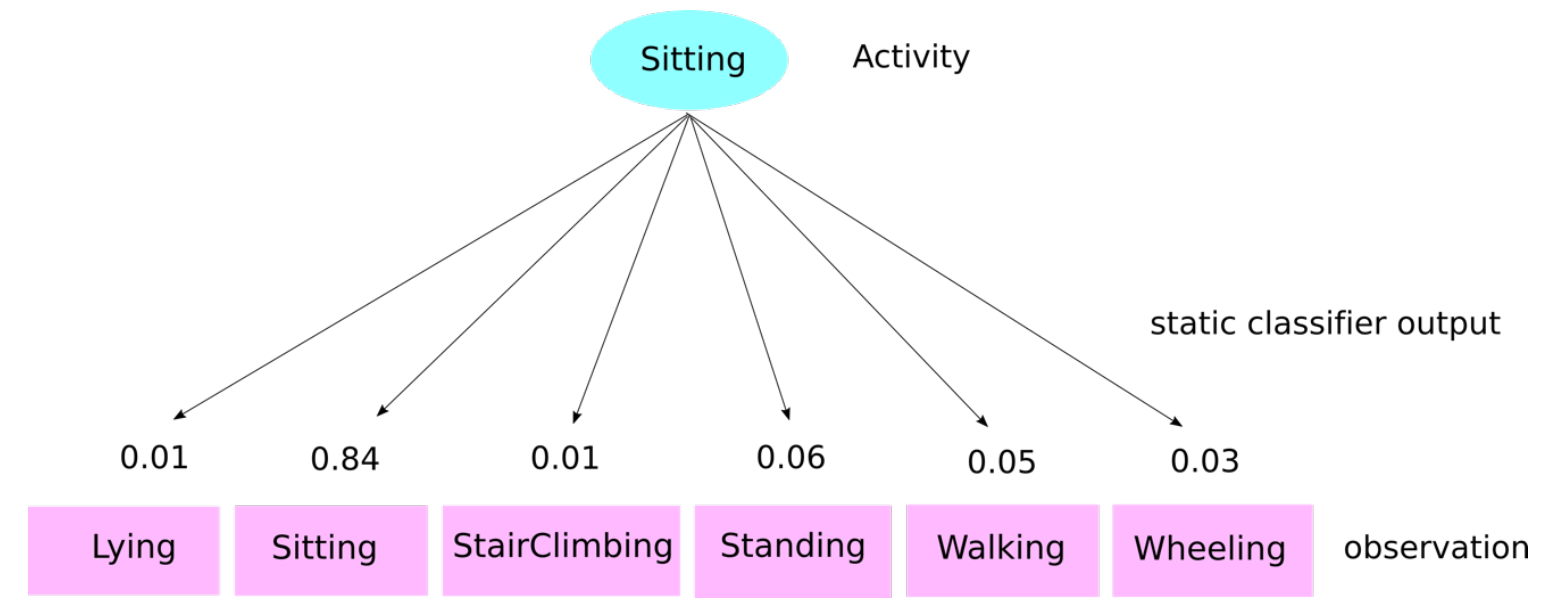

Figure 11. HMM emission probability.

Observation or emission probabilities were the results of static classifiers as shown in Figure 11. Although, static classifiers provide the HMM with a sequence of observations which correlate with the correct activity, it is not best to directly infer the 
activities/hidden states from those observations because they are prone to error due to the limits of static classification as discussed before.

We were able to construct the expected observation probabilities using known clips of data. The probabilities are modelled using guassian distributions. The mean of each gaussian was found by observing the mean probability for each predicted state over the set of all samples from a given true state, proving a distribution of means similar to the output of Figure 11. A constant standard deviation $\sigma=0.05$ was assigned to all emission distributions for simplicity, as the results are robust to a large variation in standard deviations. To test our models, we first made probabilistic predictions from the static classifiers and then used them as the input for our HMM to infer the most likely occurring activity. 

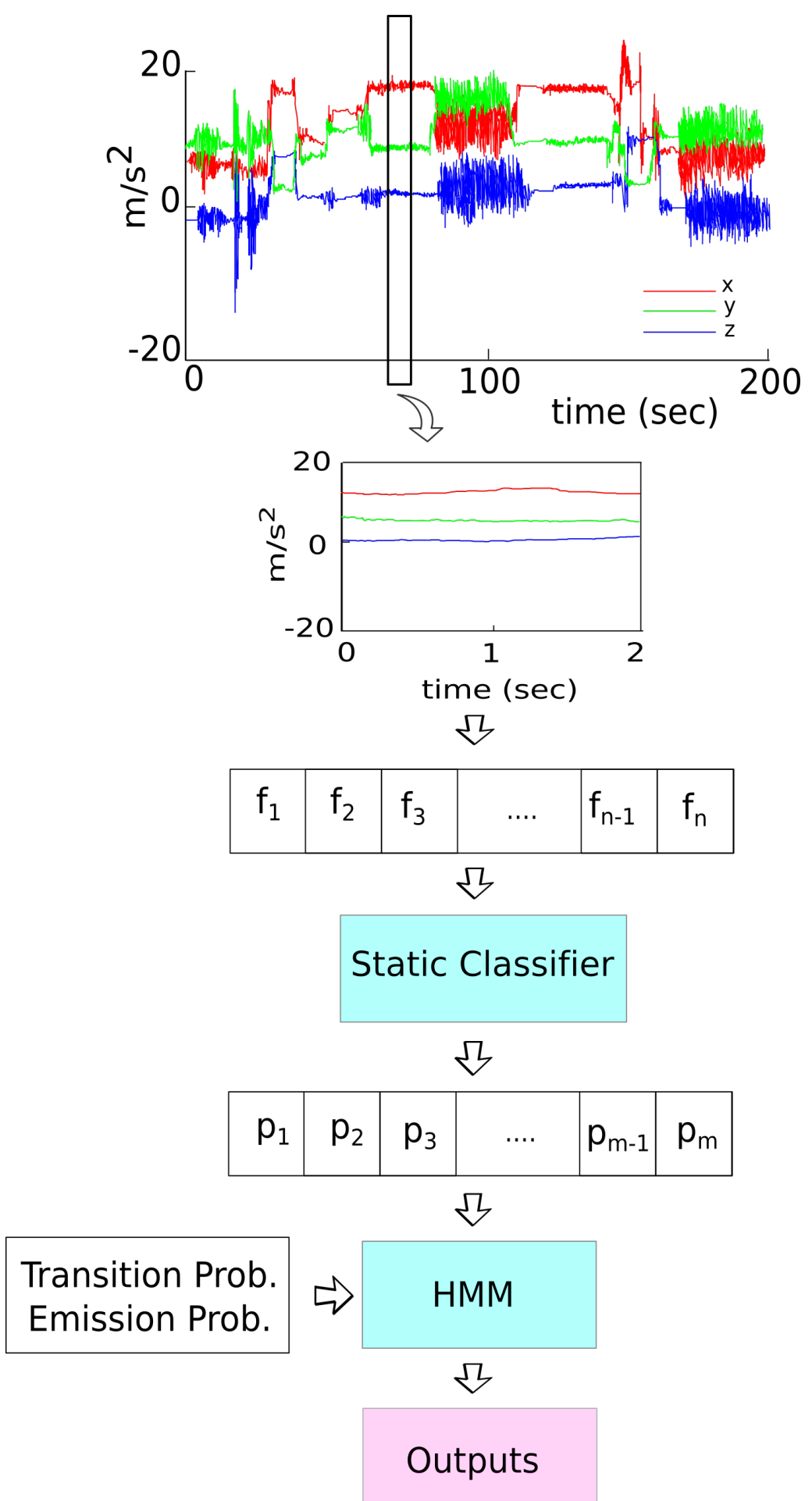

Figure 12. Classification process using hidden Markov models. 


\section{Within-Subject Cross Validation}

Cross validation is used to assess the accuracy of the model. In this study, we use within-subject cross validation and subject-wise cross validation to explore the influence of training context on classification accuracy. We applied cross validation technique on both static classification and HMM classification.

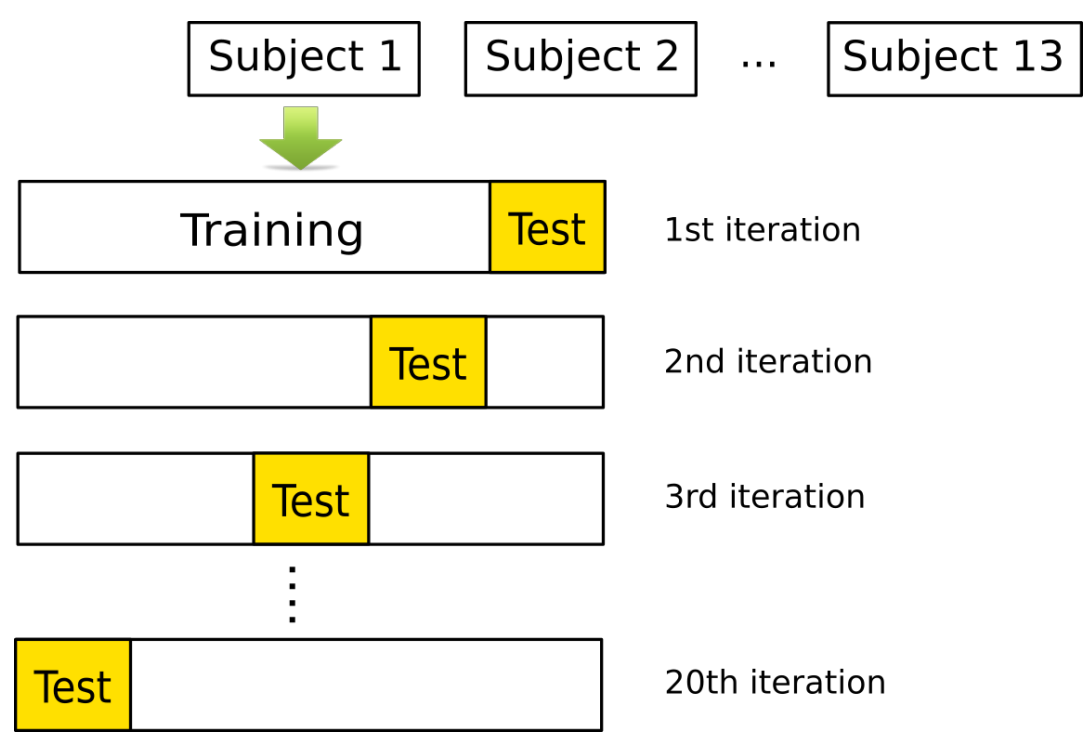

Figure 13. Within-subject 20-fold cross validation technique.

In within-subject cross validation, each subject's data is trained and tested individually using a 20-fold cross validation technique. Figure 13 demonstrates withinsubject 20 -fold cross validation. For each subject, the original dataset is partitioned into 20 equal-sized subsets. Of the 20 subsets, a classifier is trained on 19 of the datasets, and tested on the one remaining dataset. This is repeated twenty times, and a mean accuracy is then measured. Overall accuracy is based on the mean accuracy of all the subjects' accuracy scores. 


\section{Subject-Wise Cross Validation}

For a fairer comparison when a system is not trained on an individual's particular movements, we also performed subject-wise cross validation. For this type of validation, the classifier was trained on twelve of the subjects, and tested on the one remaining subject. This is repeated thirteen times, so that each subject is tested once (Figure 14).

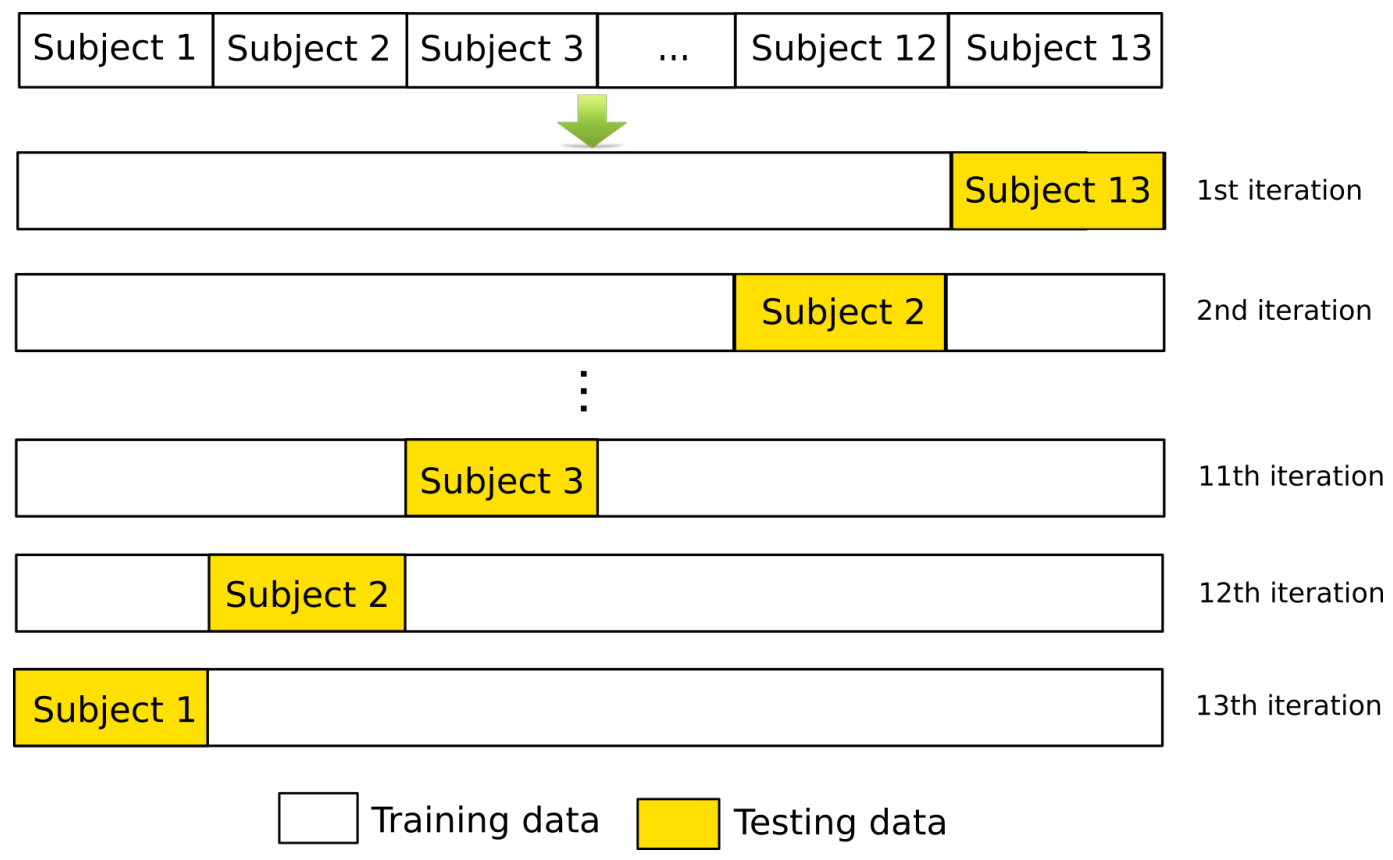

Figure 14. Subject-wise cross validation technique.

\section{Hyperparameter Optimization}

To optimize the classification algorithm's performance, a set of hyperparameters for those classifiers were chosen using a grid search of $10^{\mathrm{x}}$ where $\mathrm{x}$ is an integer between -5 and 5. We applied the following the following parameters for the classifiers. For SVM, we normalized each feature to have 0 mean and unit variance. We applied radial basis functions, giving us two hyperparameters - the soft slack variable, $C$, and the size of the Gaussian kernel, $\gamma$. Naïve Bayes had no hyperparameters. For logistic regression, 
we applied the soft slack variable, $C$ that was set to 100 and used a L1 penalty. K nearest neighbor employed with a $k$ value up to 30 . For the decision tree classifier, we applied a value of 10 corresponding to the minimum sample needed to split a node. For random forest, we applied $n$ estimators with a value up to 100 , indicating the number of decision trees to be averaged.

We also used cross validation techniques to avoid the potential for overfitting. For each iteration of the cross validation process in Figure 13 and Figure 14, we split the data into training and testing folds. On the complete training set, we used a grid search in combination with k-fold cross validation to find tune the optimum hyperparameters for each loop. The grid search will divide the training set into two halves: training fold and validation fold. After training the hyperparameters, the values will be evaluated on the validation set for each model. The model with the best hyperparameters will then be tested on the testing fold at the outer loop to evaluate the model performance. 


\section{CHAPTER IV \\ RESULTS AND DISCUSSION}

Our first goal was to augment a static standard machine learning classifier with a hidden Markov model in a laboratory setting. That is, we use in-lab data as input for the traditional, static supervised machine learning classifier, and use the results from the static classifier as input for the HMM classifier.

\section{Static Classification Results}

Using lab-acquired data, we tested the performance of the hybrid classifier for six different static classifiers-Support Vector Machine, Naïve Bayes, Regularized Logistic Regression, K Nearest Neighbor, Decision Tree, and Random Forest. Using withinsubject 20 -fold cross validation technique, we were able to predict the activity with $86.3 \%$ accuracy. The highest accuracy was from the random forest classifier followed by the support vector machine (SVM) classifier at $83.7 \%$. Overall accuracies from each classifier are presented in Table 5. The in-lab activity achieved an overall precision of $83.27 \%$ and an overall recall of $84.13 \%$. Lying was the most accurately predicted with $93.62 \%$ precision followed by wheeling at $91.30 \%$ and walking at $90.53 \%$. Table 6 illustrates the precision and recall numbers for multiple static machine learning classifiers and HMM classifiers. 
The lower accuracies were the result of misclassification of similar physical activities. The classification matrix, also known as the confusion matrix, shows the performance of the classification model (Table 8). The model tends to confuse between sitting and wheeling, and walking and stair climbing. In general, although the behaviors are quite, these particular pairs of activities are difficult to distinguish distinct due to the movement of the accelerometers on the waist, and thus the observed sensor readings, are quite similar.

For a better indication of the accuracy of our model when applied to new individuals (ones for which it has not been trained), we used subject-wise cross validation. Because individual subjects move in unique ways, especially among people with varying degrees of spinal cord injury, the overall accuracy was, as expected, lower than within-subject cross validation. The subject-wise cross validation was $63.1 \%$ accuracy (Table 5). The SVM classifier achieved the highest accuracy followed by random forest classifier. Table 9 illustrates the precision and recall numbers acquired from the SVM classifier using subject-wise cross validation.

\section{HMM Classification Results}

Using in-lab data, we trained HMM classifiers with the output probabilities of the static classifier, and tested with the test dataset. With the within-subject cross validation technique, the accuracy improved from $86.3 \%$ to $88.9 \%$. The hybrid model of random forest and HMM classifier produced 2.6\% higher accuracy compared to the one using random forest alone. We were able to accurately predict the activity with an overall precision of $87.2 \%$ and a recall of $86.9 \%$. The precision increased by $3.9 \%$ while the 
recall increased by $2.8 \%$. Each activity achieved higher accuracy compared to the prior classification. Table 7 lists the precision and recall of all activities for random forest with HMM. Stair climbing was accurately predicted with the lowest $64.29 \%$ precision using random forest alone. The result was expected, as stair climbing is easily misclassified with walking. HMM, on the other hand, correctly reclassified the activity as stair climbing and improved the accuracy to $82.4 \%$.

In subject-wise cross validation, a hybrid SVM and HMM model gave the highest accuracy of $64.3 \%$. We were able to increase the overall accuracy by $1.2 \%$, as compared to the model using only SVM. Overall recall moved from $56.78 \%$ to $62.75 \%$. The result shows the temporal power of HMM in reclassifying the activities that static classifiers fail to correctly recognize.

\begin{tabular}{|l|c|c|c|c|}
\cline { 2 - 5 } \multicolumn{1}{c|}{} & \multicolumn{2}{c|}{ Within-subject 20-fold } & \multicolumn{2}{c|}{ Subject-wise } \\
\cline { 2 - 5 } \multicolumn{1}{c|}{} & $\begin{array}{c}\text { Static } \\
\text { classifier }\end{array}$ & $\begin{array}{c}\text { HMM with } \\
\text { static classifier }\end{array}$ & $\begin{array}{c}\text { Static } \\
\text { classifier }\end{array}$ & $\begin{array}{c}\text { HMM with } \\
\text { static classifier }\end{array}$ \\
\hline $\begin{array}{l}\text { Support Vector } \\
\text { Machine }\end{array}$ & $83.7 \%$ & $86.5 \%$ & $63.1 \%$ & $64.3 \%$ \\
\hline Naïve Bayes & $82.3 \%$ & $82.9 \%$ & $56.1 \%$ & $57.5 \%$ \\
\hline $\begin{array}{l}\text { Regularized } \\
\text { Logistic Regression }\end{array}$ & $83 \%$ & $85.7 \%$ & $61.8 \%$ & $60.5 \%$ \\
\hline $\begin{array}{l}\text { K Nearest } \\
\text { Neighbors }\end{array}$ & $71 \%$ & $76.9 \%$ & $50.3 \%$ & $55.7 \%$ \\
\hline Decision Tree & $82.5 \%$ & $83.6 \%$ & $49.9 \%$ & $54.8 \%$ \\
\hline Random Forest & $86.3 \%$ & $88.9 \%$ & $60 \%$ & $62 \%$ \\
\hline
\end{tabular}

Table 5. Overall accuracy for multiple generative and discriminative classifiers. 


\begin{tabular}{|c|c|c|c|c|c|c|c|}
\hline & \multicolumn{6}{|c|}{ Classified Activity (by Random Forest) } \\
\hline & & Lying & Sitting & Wheeling & Standing & Walking & Stair Climbing \\
\hline \multirow{6}{*}{ 总 } & Lying & $93.62 \%$ & $2.66 \%$ & $0.42 \%$ & $2.17 \%$ & & $1.12 \%$ \\
\hline & Sitting & $1.94 \%$ & $74.20 \%$ & $19.98 \%$ & $3.29 \%$ & $0.42 \%$ & $0.17 \%$ \\
\hline & Wheeling & $0.11 \%$ & $7.39 \%$ & $91.30 \%$ & $0.76 \%$ & $0.16 \%$ & $0.27 \%$ \\
\hline & Standing & $1.18 \%$ & $5.59 \%$ & $0.32 \%$ & $85.66 \%$ & $4.26 \%$ & $2.99 \%$ \\
\hline & Walking & $0.05 \%$ & $0.21 \%$ & $0.05 \%$ & $1.38 \%$ & $90.53 \%$ & $7.77 \%$ \\
\hline & Stair Climbing & $0.14 \%$ & $0.43 \%$ & & $5.00 \%$ & $30.14 \%$ & $64.29 \%$ \\
\hline
\end{tabular}

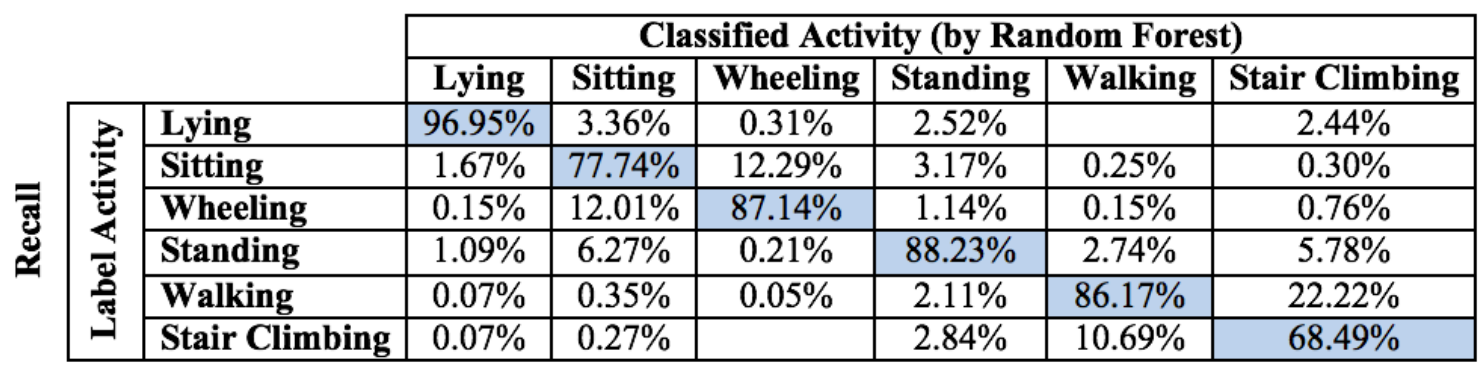

Table 6. Precision and recall numbers for random forest classifier using within-subject cross validation. Overall precision is $83.3 \%$ and recall is $84.1 \%$.

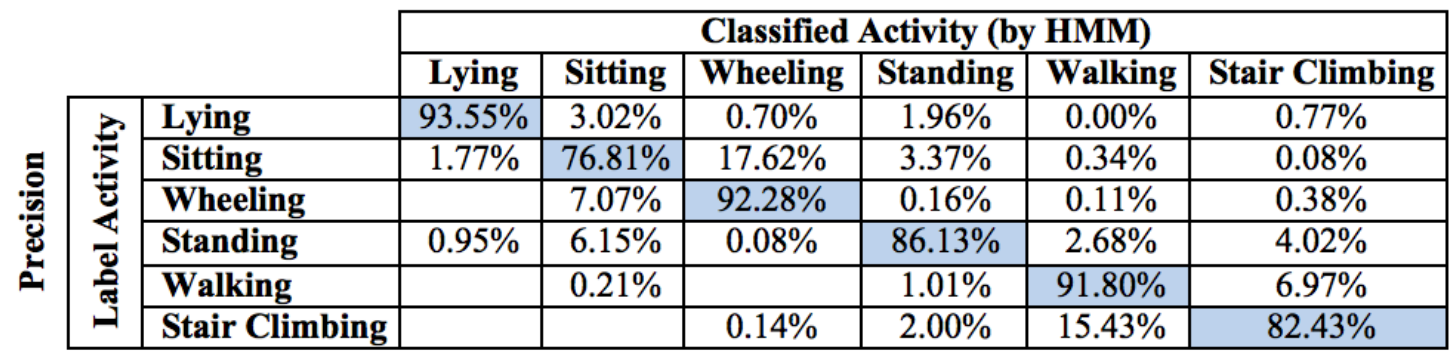

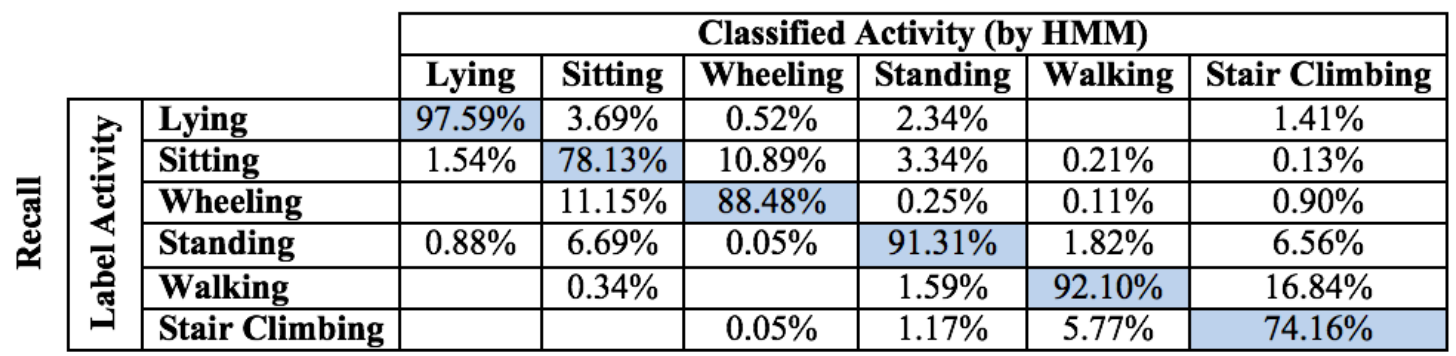

Table 7. Precision and recall numbers for HMM classifier using within-subject cross validation. Overall precision is $87.2 \%$ and recall is $86.9 \%$. 


\begin{tabular}{|c|c|c|c|c|c|c|c|}
\hline & \multicolumn{6}{|c|}{ Classified Activity (by Random Forest) } \\
\hline & & Lying & Sitting & Wheeling & Standing & Walking & Stair Climbing \\
\hline \multirow{6}{*}{ 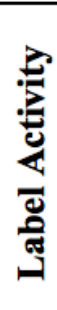 } & Lying & 1335 & 38 & 6 & 31 & 0 & 16 \\
\hline & Sitting & 23 & 880 & 237 & 39 & 5 & 2 \\
\hline & Wheeling & 2 & 136 & 1680 & 14 & 3 & 5 \\
\hline & Standing & 15 & 71 & 4 & $\mathbf{1 0 8 7}$ & 54 & 38 \\
\hline & Walking & 1 & 4 & 1 & 26 & 1701 & 146 \\
\hline & Stair Climbing & 1 & 3 & 0 & 35 & 211 & 450 \\
\hline
\end{tabular}

\begin{tabular}{|c|c|c|c|c|c|c|c|}
\hline & & \multicolumn{7}{|c|}{ Classified Activity (by HMM) } \\
\hline & & Lying & Sitting & Wheeling & Standing & Walking & Stair Climbing \\
\hline \multirow{3}{*}{} & Lying & $\mathbf{1 3 3 4}$ & 43 & 10 & 28 & 0 & 11 \\
\cline { 2 - 8 } & Sitting & 21 & $\mathbf{9 1 1}$ & 209 & 40 & 4 & 1 \\
\cline { 2 - 8 } & Wheeling & 0 & 130 & $\mathbf{1 6 9 8}$ & 3 & 2 & 7 \\
\cline { 2 - 8 } & Standing & 12 & 78 & 1 & $\mathbf{1 0 9 3}$ & 34 & 51 \\
\cline { 2 - 8 } & Walking & 0 & 4 & 0 & 19 & $\mathbf{1 7 2 5}$ & 131 \\
\cline { 2 - 8 } & Stair Climbing & 0 & 0 & 1 & 14 & 108 & $\mathbf{5 7 7}$ \\
\hline
\end{tabular}

Table 8. Classification matrix of random forest and HMM classifier using within-subject 20 -fold cross validation.

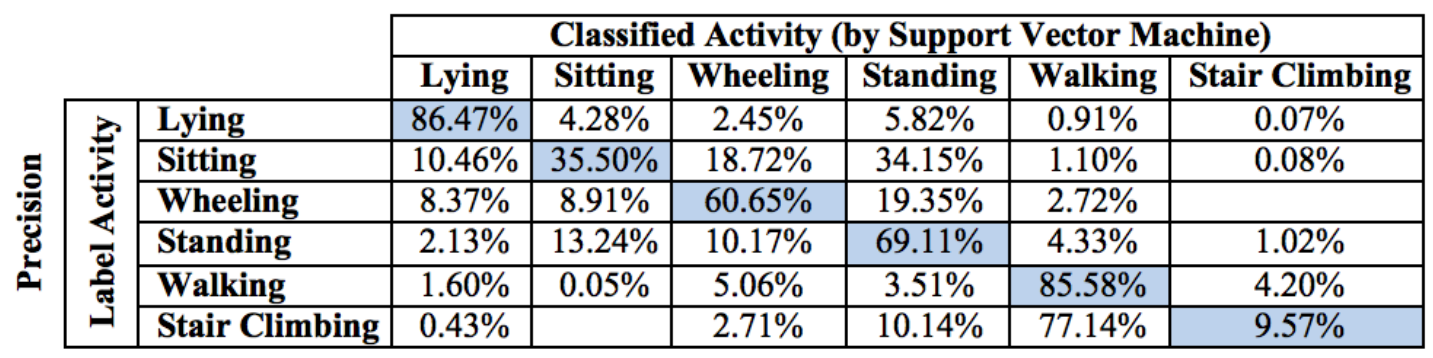

\begin{tabular}{|c|c|c|c|c|c|c|c|}
\hline & \multicolumn{6}{|c|}{ Classified Activity (by Support Vector Machine) } \\
\hline & & Lying & Sitting & Wheeling & Standing & Walking & Stair Climbing \\
\hline \multirow{6}{*}{$\overline{\widetilde{్}}$} & Lying & $78.49 \%$ & $7.48 \%$ & $2.17 \%$ & $4.47 \%$ & $0.57 \%$ & $0.62 \%$ \\
\hline & Sitting & $7.89 \%$ & $51.66 \%$ & $13.74 \%$ & $21.80 \%$ & $0.57 \%$ & $0.62 \%$ \\
\hline & Wheeling & $9.80 \%$ & $20.12 \%$ & $69.06 \%$ & $19.16 \%$ & $2.19 \%$ & \\
\hline & Standing & $1.72 \%$ & $20.61 \%$ & $7.98 \%$ & $47.20 \%$ & $2.41 \%$ & $8.07 \%$ \\
\hline & Walking & $1.91 \%$ & $0.12 \%$ & $5.88 \%$ & $3.55 \%$ & $70.56 \%$ & $49.07 \%$ \\
\hline & Stair Climbing & $0.19 \%$ & & $1.18 \%$ & $3.82 \%$ & $23.69 \%$ & $41.61 \%$ \\
\hline
\end{tabular}

Table 9. Precision and recall numbers for SVM classifier using subject-wise cross validation. Overall precision is $57.81 \%$ and recall is $56.78 \%$. 


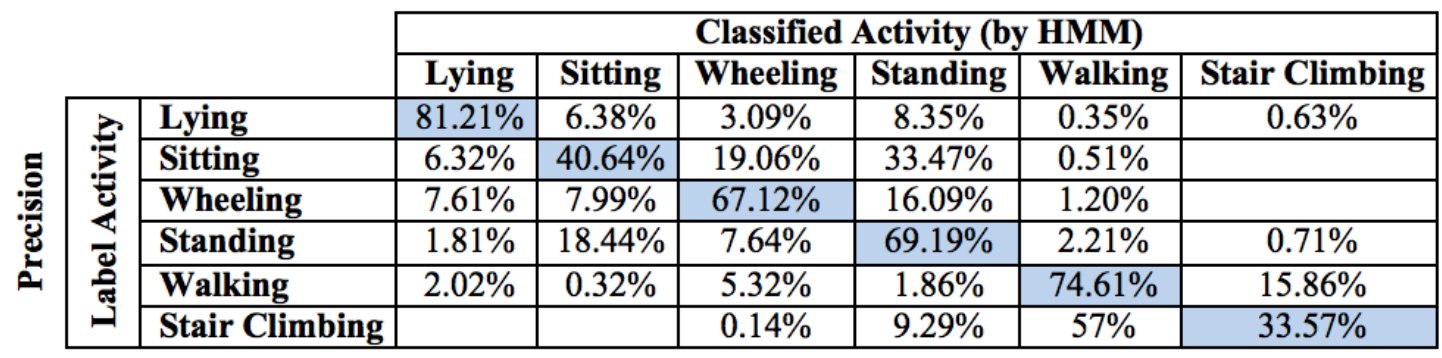

\begin{tabular}{|c|c|c|c|c|c|c|c|}
\hline & \multicolumn{6}{|c|}{ Classified Activity (by HMM) } \\
\hline & & Lying & Sitting & Wheeling & Standing & Walking & Stair Climbing \\
\hline \multirow{6}{*}{ 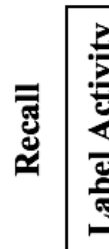 } & Lying & $80.75 \%$ & $9.48 \%$ & $2.58 \%$ & $6.65 \%$ & $0.27 \%$ & $1.63 \%$ \\
\hline & Sitting & $5.23 \%$ & $50.21 \%$ & $13.27 \%$ & $22.18 \%$ & $0.32 \%$ & \\
\hline & Wheeling & $9.76 \%$ & $15.31 \%$ & $72.52 \%$ & $16.54 \%$ & $1.18 \%$ & \\
\hline & Standing & $1.60 \%$ & $24.38 \%$ & $5.70 \%$ & $49.05 \%$ & $1.50 \%$ & $1.63 \%$ \\
\hline & Walking & $2.65 \%$ & $0.63 \%$ & $5.87 \%$ & $1.96 \%$ & $75.30 \%$ & $54.08 \%$ \\
\hline & Stair Climbing & & & $0.06 \%$ & $3.63 \%$ & $21.43 \%$ & $42.65 \%$ \\
\hline
\end{tabular}

Table 10. Precision and recall numbers for HMM classifier using subject-wise cross validation. Overall precision is $61.06 \%$ and recall is $62.75 \%$.

\begin{tabular}{|c|c|c|c|c|c|c|c|}
\cline { 3 - 8 } \multicolumn{2}{c|}{} & \multicolumn{6}{c|}{ Classified Activity (by Support Vector Machine) } \\
\cline { 3 - 8 } \multicolumn{2}{c|}{} & Lying & Sitting & Wheeling & Standing & Walking & Stair Climbing \\
\hline \multirow{3}{*}{} & Lying & $\mathbf{1 2 3 3}$ & 61 & 35 & 83 & 13 & 1 \\
\cline { 2 - 8 } & Sitting & 124 & $\mathbf{4 2 1}$ & 222 & 405 & 13 & 1 \\
\cline { 2 - 8 } & Wheeling & 154 & 164 & $\mathbf{1 1 1 6}$ & 356 & 50 & 0 \\
\cline { 2 - 8 } & Standing & 27 & 168 & 129 & $\mathbf{8 7 7}$ & 55 & 13 \\
\cline { 2 - 8 } & Walking & 30 & 1 & 95 & 66 & $\mathbf{1 6 0 8}$ & 79 \\
\cline { 2 - 8 } & Stair Climbing & 3 & 0 & 19 & 71 & 540 & $\mathbf{6 7}$ \\
\hline
\end{tabular}

\begin{tabular}{|c|c|c|c|c|c|c|c|}
\hline & & \multicolumn{6}{|c|}{ Classified Activity (by HMM) } \\
\hline & & Lying & Sitting & Wheeling & Standing & Walking & Stair Climbing \\
\hline \multirow{6}{*}{ 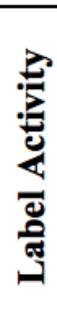 } & Lying & 1158 & 91 & 44 & 119 & 5 & 9 \\
\hline & Sitting & 75 & 482 & 226 & 397 & 6 & 0 \\
\hline & Wheeling & 140 & 147 & 1235 & 296 & 22 & 0 \\
\hline & Standing & 23 & 234 & 97 & 878 & 28 & 9 \\
\hline & Walking & 38 & 6 & 100 & 35 & 1402 & 298 \\
\hline & Stair Climbing & 0 & 0 & 1 & 65 & 399 & 235 \\
\hline
\end{tabular}

Table 11. Classification matrix of SVM and HMM classifier using subject-wise cross validation. 


\section{Discussion}

By using HMMs to correct misclassifications produced by static classifiers in activity recognition, we were able to increase the prediction accuracy by $2.6 \%$ when using within-subject cross validation. With subject-wise cross validation, the accuracy increases by $1.2 \%$. This confirms our hypothesis of increased accuracy in activity recognition when using HMMs to supplement a static classifier.

Our results have aligned with other studies regarding the use of hidden Markov models with increased accuracy in activity recognition rather than using static classifiers alone (Olguín \& Pentland, 2006; Lester, Choudhury, Kern, Borriello, \& Hannaford, 2005). Antos, Albert and Kording (2014) have also shown the improved classification accuracy in activity and location tracking by integrating static classifiers into HMMs to smooth out temporal irregularities.

Our prediction improvement, however, was subtle because we dealt with activities that are almost undistinguishable. As shown in confusion matrix in Table 8 and Table 11, the model mostly confuses between sitting and wheeling, and walking and stair climbing. In general, sitting and wheeling are difficult to differentiate when one subject had to use joystick-controlled wheelchair during data collection, which makes sitting and wheeling on a wheelchair are relatively similar on the accelerometer readings. Some patients walk and climb up the stair at the same slow pace, which made it hard for the classification model to recognize between walking and stair climbing. If we did not distinguish those activities, we were able to achieve up to approximately $96 \%$. Beyond the concern of these movement variations, the performance of static classifiers could also affect the overall 
accuracy. If static classifiers perform very poorly on a consistent basis, the HMM has little useful information to work with. In order to increase the overall effectiveness and applicability of this program more work will likely need to be done at the static classification stage.

With results and observations that we have gathered, there are many directions this project could be taken in the future. For example, smart phones have been used activity recognition purposes using HMMs (Antos, Albert, \& Kording, 2014). In this particular example, the models were trained from different locations on the body, something we could consider doing in the future. Alternatively, there have been studies which use multiple accelerometers at once at different locations on the body with the hope that the accelerometers could collectively provide better data than the use of a single accelerometer (Olguín \& Pentland, 2006). On the other hand, instead of training our model with incomplete spinal cord injury patients only, we would have trained our model to detect each form of activity that is unique to the population of each physical therapy group, such as incomplete spinal cord injury and Parkinson's disease as these individuals move in similar ways compared to others within their group, but not necessarily others. 


\section{CHAPTER V \\ CONCLUSION}

In this research study, we developed a model that integrates static classifiers with a hidden Markov model (HMM). The objective of this approach is to improve accuracy by leveraging the individual, discriminative ability of static classifiers and the ability of HMMs to use temporal relationships of classified activities. Results show that augmenting static classifiers with HMMs, in general, gives better classification accuracy over static machine learning classifiers alone.

Through the improved prediction accuracy, this hybrid approach brings more advanced activity recognition closer to clinical application. People with mobility impairments, such as those with incomplete spinal cord injury, have an extensive need for physical therapy to improve recovery outcomes. Assessing the impact of therapy at home benefits from the type of data that can be collected through activity recognition. Wearable devices are capable of measuring patient outcomes objectively, conveniently, and continuously compared to more traditional assessment tools-like clinical visits and home journaling. In this way we hope this work, and activity recognition in general, will lead to better therapeutic interventions and patient outcomes. 


\section{REFERENCES}

Albert, M. V., Azeze, Y., Courtois, M., \& Jayaraman, A. (2016). In-lab versus at-home activity recognition in ambulatory subjects with incomplete spinal cord injury. Transactions on Neural Systems and Rehabilitation Engineering (submitted)

Albert M.V., Kording K.P., Herrmann M., Jayaraman A. (2012) Fall classification by machine learning using mobile phones. PLoS ONE 7(5): e36556

Albert, M. V., Toledo, S., Shapiro, M., \& Koerding, K. (2012). Using mobile phones for activity recognition in Parkinson's patients. Frontiers in Neurology, 3(158). http://doi.org/10.3389/fneur.2012.00158

Anderson, K. D. (2004). Targeting Recovery: Priorities of the Spinal Cord-Injured Population. Mary Ann Liebert, Inc., 21, 1371-1383.

Antos, S. A., Albert, M. V., \& Kording, K. P. (2014). Hand, belt, pocket or bag: Practical activity tracking with mobile phones. Journal of Neuroscience Methods, 231, 2230. http://doi.org/10.1016/j.jneumeth.2013.09.015

Arif, M., \& Kattan, A. (2015). Physical Activities Monitoring Using Wearable Acceleration Sensors Attached to the Body. PLoS ONE, 10(7), e0130851. http://doi.org/10.1371/journal.pone.0130851

Chiauzzi, E., Rodarte, C., \& DasMahapatra, P. (2015). Patient-centered activity monitoring in the self-management of chronic health conditions. BMC Medicine, 13. http://doi.org/10.1186/s12916-015-0319-2

Fosler-Lussier, E. (1998). Markov Models and Hidden Markov Models: A Brief Tutorial. International Computer Science Institute, Berkeley.

Gaikwad, K. (2012). HMM Classifier for Human Activity Recognition. Computer Science \& Engineering: An International Journal, 2(4), 27-36. http://doi.org/10.5121/cseij.2012.2403

Ganapathiraju, A., Hamaker, J., \& Picone, J. (2000). Hybrid SVM/HMM Architectures for Speech Recognition. In in Speech Transcription Workshop (pp. 504-507).

Introduction to Support Vector Machines - OpenCV 2.4.12.0 documentation. (n.d.). Retrieved March 10, 2016, from 
http://docs.opencv.org/2.4/doc/tutorials/ml/introduction_to_svm/introduction_t o_svm.html

Kim, E., Helal, S., \& Cook, D. (2010). Human Activity Recognition and Pattern Discovery. IEEE Pervasive Computing, 9(1), 48-53. http://doi.org/10.1109/MPRV.2010.7

Kooiman, T. J. M., Dontje, M. L., Sprenger, S. R., Krijnen, W. P., van der Schans, C. P., $\&$ de Groot, M. (2015). Reliability and validity of ten consumer activity trackers. BMC Sports Science, Medicine and Rehabilitation, 7(1), 1-11. http://doi.org/10.1186/s13102-015-0018-5

Kwapisz, J. R., Weiss, G. M., \& Moore, S. A. (2011). Activity Recognition Using Cell Phone Accelerometers. SIGKDD Explor. Newsl., 12(2), 74-82. http://doi.org/10.1145/1964897.1964918

Lee, J. M., Kim, Y., \& Welk, G. J. (2014). Validity of consumer-based physical activity monitors. Med Sci Sports Exerc, 46. http://doi.org/10.1249/MSS.0000000000000287

Lester, J., Choudhury, T., \& Borriello, G. (2006). A Practical Approach to Recognizing Physical Activities. In K. P. Fishkin, B. Schiele, P. Nixon, \& A. Quigley (Eds.), Pervasive Computing: 4th International Conference, PERVASIVE 2006, Dublin, Ireland, May 7-10, 2006. Proceedings (pp. 1-16). Berlin, Heidelberg: Springer Berlin Heidelberg. Retrieved from http://dx.doi.org/10.1007/11748625_1

Lester, J., Choudhury, T., Kern, N., Borriello, G., \& Hannaford, B. (2005). A Hybrid Discriminative/Generative Approach for Modeling Human Activities. In Proceedings of the 19th International Joint Conference on Artificial Intelligence (pp. 766-772). San Francisco, CA, USA: Morgan Kaufmann Publishers Inc. Retrieved from http://dl.acm.org/citation.cfm?id=1642293.1642416

Najafi, B., Aminian, K., Paraschiv-Ionescu, A., Loew, F., Bula, C. J., \& Robert, P. (2003). Ambulatory system for human motion analysis using a kinematic sensor: monitoring of daily physical activity in the elderly. IEEE Transactions on Biomedical Engineering, 50(6), 711-723. http://doi.org/10.1109/TBME.2003.812189

Olguín, D. O., \& Pentland, A. (2006). Human activity recognition: Accuracy across common locations for wearable sensors. In IN PROC. 10TH INT. SYMP. WEARABLE COMPUTERS (pp. 11-13).

Patel, S., Mancinelli, C., Healey, J., Moy, M., \& Bonato, P. (2009). Using Wearable Sensors to Monitor Physical Activities of Patients with COPD: A Comparison of Classifier Performance. In Proceedings of the 2009 Sixth International Workshop on Wearable and Implantable Body Sensor Networks (pp. 234-239). Washington, DC, USA: IEEE Computer Society. http://doi.org/10.1109/BSN.2009.53 
Ravi, N., Dandekar, N., Mysore, P., \& Littman, M. L. (2005). Activity Recognition from Accelerometer Data. In Proceedings of the 17th Conference on Innovative Applications of Artificial Intelligence - Volume 3 (pp. 1541-1546). Pittsburgh, Pennsylvania: AAAI Press. Retrieved from

http://dl.acm.org/citation.cfm?id=1620092.1620107

Stone, A. A., Shiffman, S., Schwartz, J. E., Broderick, J. E., \& Hufford, M. R. (2003). Patient compliance with paper and electronic diaries. Controlled Clinical Trials, 24(2), 182 - 199. http://doi.org/http://dx.doi.org/10.1016/S0197-2456(02)00320-3

Wirz, M., Zemon, D. H., Rupp, R., Scheel, A., Colombo, G., Dietz, V., \& Hornby, T. G. (2005). Effectiveness of automated locomotor training in patients with chronic incomplete spinal cord injury: A multicenter trial. Archives of Physical Medicine and Rehabilitation, 86(4), 672-680. http://doi.org/10.1016/j.apmr.2004.08.004 
VITA

Pichleap Sok was born and raised in Phnom Penh, Cambodia. In 2013, she attended Universiti Teknologi PETRONAS, Perak, Malaysia, where she earned a Bachelor of Technology in Information and Communication Technology, with First Class Honors. From 2014 to 2016, she was awarded a Fulbright scholarship to pursue a Master's degree in Computer Science at Loyola University Chicago.

In 2012, she was selected to be one of the twelve recipients of the Google Anita Borg Memorial Scholarship Asian by demonstrating her strong interest in Computer Science, leadership, and outstanding academic record. She was also awarded a GHC scholarship grant to participate in the 2015 Grace Hopper Celebration for Women in Computing in Houston, Texas.

While at Loyola, she was honored with Dijkstra Award for Outstanding Academic Achievement from the Department of Computer Science. The award generally recognizes the single student with top academic performance across all computer science degrees. She and her team won the first place at Techweek Chicago Hackathon in 2015 and the third place in INTXHACK Finals at the 2015 Internet and Television Expo (INTX) in Chicago, Illinois. 